\title{
A Computational Theory of Normative Positions
}

\author{
MAREK SERGOT \\ Imperial College of Science, Technology and Medicine, London
}

\begin{abstract}
The Kanger-Lindahl theory of normative positions attempts to use a combination of deontic logic (the logic of obligation and permission) and a logic of action/agency to give a formal account of obligations, duties, rights, and other complex normative concepts. This paper presents a generalisation and further development of this theory, together with methods for its automation and application to practical examples. The resulting theory is intended to be applied in the representation and analysis of laws, regulations, and contracts, in the specification of aspects of computer systems, in multi-agent systems, and as a contribution to the formal theory of organisations. Particular attention is paid to representations at varying levels of detail and the relationships that hold between them. The last part presents Norman-G, an automated support system intended to facilitate application of the theory to the analysis of practical problems, with a small example to illustrate its use.
\end{abstract}

Categories and Subject Descriptors: F.4.1 [Mathematical Logic and Formal Languages]: Mathematical Logic-Modal logic; I.2.4 [Artificial Intelligence]: Knowledge Representation Formalisms and Methods-Modal logic; I.2.1 [Artificial Intelligence]: Applications and Expert Systems - Law; D.2.1 [Software Engineering]: Requirements/Specifications

General Terms: Algorithms, Theory

Additional Key Words and Phrases: Deontic logic, normative systems, logic of action, logic of agency, theory of duties and rights

\section{INTRODUCTION}

The general topic of this paper is the formal representation of agents' obligations, permissions, duties and rights, and other complex normative relations such as entitlement, authorisation, responsibility. Such concepts are usually discussed within the context of law and legal relations. Hohfeld, for instance, whose work [Hohfeld $1913]$ is still often given as the standard reference to this field, referred to them as the 'fundamental legal conceptions'. It is important to establish, however, that these are not, in fact, exclusively legal concepts, but characteristic of all forms of regulated and organised agent interaction, legal or non-legal, formal or informal. Although the work described here is intended to be employed in the formal representation of laws and regulations and legal contracts-Allen and Saxon, for

A shorter version of the middle part of this paper appears under the title 'Normative Positions' in Norms, Logics and Information Systems, P. McNamara and H. Prakken, Eds. IOS Press, Amsterdam, 1998.

Author's address: M.J. Sergot, Department of Computing, Imperial College of Science, Technology and Medicine, 180 Queen's Gate, London SW7 2BZ, UK. mjs@doc.ic.ac.uk.

Permission to make digital/hard copy of all or part of this material without fee for personal or classroom use provided that the copies are not made or distributed for profit or commercial advantage, the ACM copyright/server notice, the title of the publication, and its date appear, and notice is given that copying is by permission of the ACM, Inc. To copy otherwise, to republish, to post on servers, or to redistribute to lists requires prior specific permission and/or a fee.

(C) 20TBD ACM 1529-3785/TBD/TBD $\$ 5.00$ 
example, have long argued that proper attention to the Hohfeldian concepts is essential for legal knowledge representation (see e.g. [Allen and Saxon 1986; 1993]) — it is also intended to be applied in other areas, such as the specification of aspects of computer systems (see e.g. [Jones and Sergot 1992; 1993]), and as a contribution to the formal theory of organisations, in the analysis of notions such as responsibility, authorisation and delegation.

In the literature on multi-agent systems, the notion of commitment, of an agent $a$ to another agent $b$ that such-and-such a state of affairs $F$ shall obtain or that such-and-such an action $\alpha$ will be performed, features prominently, particularly in discussions of social aspects, such as arise in dealing with co-ordinated action and joint planning. Although it must be noted that the term 'commitment' is used in multi-agent systems to refer to a wide variety of different concepts (see e.g. the various senses in which it is used in [Jennings 1993]), one common usage is the sense of an obligation or duty directed from one agent to another. This is the sense, for example, in which Shoham [1991; 1993] uses the term when presenting the logical underpinnings of his Agent-Oriented Programming (though not necessarily in the programming language Agent-0 itself). Recently, several authors, e.g. [Singh 1998; 1999; Colombetti 1999; 2000], have argued that the notion of commitment, in the sense of a directed obligation or duty, provides a much sounder basis for the semantics of agent communication languages (ACLs) than the internal mentalistic states in terms of which most ACL semantics are attempted at present.

This then is the general area of study. The specific topic of this paper is a generalisation and further development of the Kanger-Lindahl theory of normative positions, together with methods for its automation. The theory of normative positions is generally regarded as the most comprehensive and best developed attempt to formalize distinctions such as Hohfeld's. It originates in Stig Kanger's pioneering attempts to apply modal logic - primarily deontic logic (the logic of obligation and permission) and the logic of action/agency - to the representation of legal/normative relations. The methods are presented in [Kanger 1971; 1985; Kanger and Kanger 1966] with a more general account of related issues in [Kanger 1972]. As described later in the paper, Lars Lindahl [1977] developed Kanger's account in several important respects, providing also a commentary on the relationships to Hohfeld's work and the jurisprudential tradition within which it falls. Ingmar Pörn [1977] applied similar techniques to the study of what he called 'control' and 'influence' relations in social interactions. For further discussion of the theory and some of its features, see e.g. [Talja 1980; Makinson 1986; Lindahl 1992; Jones and Sergot 1993; Herrestad and Krogh 1995; Herrestad 1996; Krogh 1997]. Jones and Sergot $[1992 ; 1993]$ present a modified version of the Kanger-Lindahl theory and discuss how it may be applied to a problem in computer science concerning the specification of access control to databases of sensitive (medical) information.

A distinctive feature of the Kanger-Lindahl-Pörn approaches is a method for mapping out in a systematic and exhaustive fashion the complete space of all logically possible (normative, control, influence) relations between two agents with respect to some given act-type. These are the (normative, control, influence) 'positions'. For example, Hohfeld identified four distinct legal/normative relations that could hold between any two agents with respect to some given act type. Some examples are given later in Section 2. Kanger's systematic, formal analysis yielded 26 distinct 
such relations or 'normative positions'. Lindahl's subsequent analysis produced 35 of the same basic kind as Kanger's and 127 if a more precise set of possible relationships is considered instead. Section 3 discusses the methods in more detail and explains why there are more possibilities still than are accounted for in Lindahl's version.

The present paper generalises the Kanger-Lindahl accounts in the following respects:

(1) The generalised theory deals with interaction between any number of agents, not just two, including 'ought-to-be' statements where no agent is specified.

(2) The Kanger-Lindahl-Pörn theories deal with act expressions of the form 'agent $x$ brings it about that $F$ '. The generalised theory allows any number of such act expressions in any combination, and allows (in principle at least) compound acts, that is to say, boolean compounds of propositions in the scope of the 'brings it about' operator.

(3) There is complete separation of the method of generating 'positions' from properties of the underlying modal logics. This means that the theory does not rely on any in-built assumptions about the specific deontic or action logics employed. It also means that a richer combination of modalities can be used, to represent more complex notions.

(4) Special attention is given to practical applicability of the theory, which raises a set of questions not previously investigated. The middle part of the paper deals with representations at varying levels of detail and the relationships that hold between them. This is so that the analysis of any practical example can be conducted to whatever level of detail is desired through a process of progressive refinement. There is also a notion of independence of acts which is essential for practical application.

(5) It is possible to give an abstract account of the structure of 'positions', which reduces, and in the most common cases eliminates altogether, the need for modallogical theorem provers in the implementation of automated support tools. The last part of the paper presents Norman-G, an automated system intended to facilitate application of the theory to the analysis of practical problems. Norman-G employs a graphical interface designed so that its use does not pre-suppose familiarity with the underlying theory.

One of the aims of this paper is to illustrate the kinds of nuances and distinctions that can be articulated in the (extended) Kanger-Lindahl framework and to indicate how they can arise in practical settings. However, it must be understood that we are here presenting a component of a formal treatment of duty and right, and not a complete theory of all aspects of these complex concepts. There are welldocumented limitations of the Kanger-Lindahl framework, in particular in regard to its treatment of the feature Hohfeld called '(legal) power', also referred to sometimes as 'legal capacity' or 'competence'. See e.g. [Makinson 1986; Lindahl 1992] for a discussion of these points, and [Jones and Sergot 1996] for a proposed formal account of 'power'. For a more complete theory of duty and right, the methods presented in this paper need to be augmented: with a treatment of 'power', with temporal constructs, and with a richer set of action concepts, at the very least. These extensions will not be developed in this paper. 

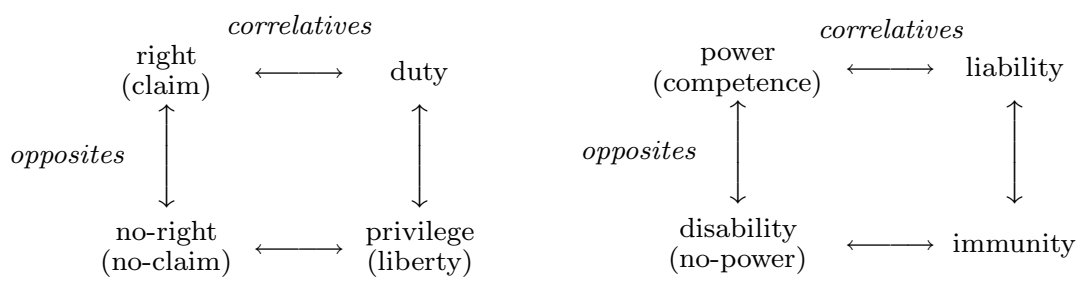

Fig. 1. Hohfeld's 'fundamental legal conceptions'

\section{PRELIMINARY DISCUSSION}

Hohfeld's seminal work [1913] is still often taken as the starting point for much that is written in this field. It identified two groups of four concepts with various relationships between them, as summarized in Figure 1. Right and duty are 'correlatives' in the sense that when $x$ has a right (a 'claim-right') against $y$ that $F$ (be done by $y$ ) then $y$ owes a duty to $x$ that $F$ (be done by $y$ ); and conversely. The relationships may be summarised semi-formally by the following scheme, adapted from [Lindahl 1977]:

$$
\begin{aligned}
\operatorname{Right}(x, y, F) & \leftrightarrow \operatorname{Duty}(y, x, F) \\
\operatorname{Right}(x, y, \operatorname{not}-F) & \leftrightarrow \operatorname{Duty}(y, x, \text { not- } F)
\end{aligned}
$$

Here not- $F$ is intended to stand for $y$ 's refraining from doing $F$. Of course it remains to explain how this notion of refraining is to be represented formally; this is one of the features of Kanger's framework.

Duty and privilege (some authors prefer 'liberty') are 'opposites' in the Hohfeldian scheme in the sense that $x$ has a privilege/liberty from $y$ with respect to $F$ when $x$ does not owe a duty to $y$ to refrain from $F$, and $x$ has a privilege/liberty from $y$ to refrain from $F$ when $x$ does not owe a duty to $y$ that $F$ (be done by $x$ ). In the semi-formal notation these relationships may be summarised as follows:

$$
\begin{aligned}
\text { Privilege }(x, y, F) & \leftrightarrow \neg D \text { uty }(x, y, \operatorname{not}-F) \\
\text { Privilege }(x, y, \operatorname{not}-F) & \leftrightarrow \neg D \text { uty }(x, y, F)
\end{aligned}
$$

Similarly, right/no-right and no-right/privilege are also opposite and correlative pairs, respectively, in the Hohfeldian scheme, in the following sense:

$$
\begin{aligned}
\operatorname{Right}(x, y, F) & \leftrightarrow \neg N o \text {-right }(x, y, F) \\
\operatorname{Right}(x, y, \text { not- } F) & \leftrightarrow \neg N o \text {-right }(x, y, \text { not- } F) \\
\text { No-right }(x, y, F) & \leftrightarrow \operatorname{Privilege}(y, x, \operatorname{not}-F) \\
\text { No-right }(x, y, \text { not- } F) & \leftrightarrow \operatorname{Privilege}(y, x, F)
\end{aligned}
$$

One can see already, however, as pointed out in [Lindahl 1977, pp26-27] and in [Kanger and Kanger 1966], that there are discrepancies in Hohfeld's account: the right/duty and no-right/privilege correlative pairs are not exactly of the same form, and nor are the right/no-right and duty/privilege opposites. 
There is further inexactitude in Hohfeld's scheme for his second group of concepts, those on the right of the diagram in Figure 1. This second group is concerned with changes of legal/normative relations, as when it is said, for example, that $x$ has power (competence) to impose a duty on $y$ that such-and-such or to grant a privilege or right to $z$ that such-and-such. Discussion of this second set of concepts raises a new set of questions however and is beyond the scope of this paper. The second part of [Lindahl 1977] is concerned with this group of concepts. See [Jones and Sergot 1996] for an alternative account of power/competence.

For present purposes, the point is that Hohfeld's writings, and much else that has been written on these topics in legal theory, provide a wealth of examples and the beginnings of a systematic account, but are not precise enough to give a formal theory. Kanger attempted to provide such a theory by applying the formal tools of modal logic to this task.

The Kanger-Lindahl theory has a deontic logic component, an action logic component, and a method for generating the space of all logically possible positions. The language is that of propositional logic augmented with modal operators $\mathrm{O}$ (for 'obligation') and its dual P (for 'permission'), and relativised modal operators $\mathrm{E}_{a}, \mathrm{E}_{b}, \ldots$ for act expressions, where $a, b, \ldots$ are the names of individual agents. (This notation is slightly different from Kanger and Lindahl's, who use Shall and May for $\mathrm{O}$ and $\mathrm{P}$, and Do for act expressions. The notation of this paper is chosen primarily because it reduces the size of the formal expressions to be manipulated.)

An expression of the form $\mathrm{O} A$ may be read as 'it is obligatory that $A$ ' or 'it ought to be the case that $A^{\prime}$. P is the dual of $\mathrm{O}: \mathrm{P} A \stackrel{\text { def }}{=} \neg \mathrm{O} \neg A$. The expression $\mathrm{P} A$ may be read as 'it is permissible that $A$ '. The deontic logic employed by Kanger and Lindahl is - for all intents and purposes - the system usually referred to as Standard Deontic Logic (SDL). Specifically, the deontic logic employed is the smallest system containing propositional logic $(P L)$ and the following axiom schemas and rules:

$$
\begin{array}{cc}
\text { O.RE } & \frac{A \leftrightarrow B}{\mathrm{O} A \leftrightarrow \mathrm{O} B} \\
\text { O.M } & \mathrm{O}(A \wedge B) \rightarrow(\mathrm{O} A \wedge \mathrm{O} B) \\
\text { O.C } & (\mathrm{O} A \wedge \mathrm{O} B) \rightarrow \mathrm{O}(A \wedge B) \\
\text { O.P } & \neg \mathrm{O} \perp
\end{array}
$$

The names of axiom schemas and rules in this paper are based on those of [Chellas 1980]: in the now standard classification, the logic of O is a classical modal logic of type EMCP. For comparison, Standard Deontic Logic (SDL) is a normal modal logic of type $K D$, which is type $E M C P$ together with the additional rule of necessitation

$$
\text { O.RN } \frac{A}{\mathrm{O} A}
$$

or, equivalently, the axiom schema $\mathrm{O} \top$ ( $\top$ any tautology). The absence or presence of rule O.RN plays no role in the generation of normative positions: this is why we say that Kanger's choice of a deontic logic is to all intents and purposes Standard Deontic Logic. The 'deontic axiom' of Standard Deontic Logic

O.D $\quad \mathrm{O} A \rightarrow \mathrm{P} A$ 
is easily derived from O.C and O.P.

It should be noted that Standard Deontic Logic (of type KD or EMCP) has many well-known limitations as a formal theory of obligation and permission and its inadequacies are taken as the point of departure for many of the developments in the field of deontic logic. Both axioms O.M and O.C can be criticised as simplistic, for example. However, in combination with the logic of action, and in the restricted ways it is employed in the generation of normative positions, these inadequacies are relatively benign. In any case, the extended theory of normative positions to be presented in later sections is not dependent on specific choices for the deontic and action logics employed. These can be changed, as explained below.

As regards the action component, expressions of the form $\mathrm{E}_{x} A$ stand for 'agent $x$ sees to it that, or brings it about that, $A$ '. This approach to the logic of action has been extensively studied in analytical philosophy and philosophical logic but is perhaps not so familiar in Computer Science. The stit operator of [Belnap and Perloff 1988; 1992] and dstit of [Horty and Belnap 1995] are instances of the general approach that have had some exposure in the AI literature. The focus of attention is not on transitions and state changes as in most treatments of action in AI and Computer Science, but rather on the end result $A$ and the agent $x$ whose actions are responsible, in some appropriate sense, for this end result; the specific means or actions employed by agent $x$ to bring about $A$ are not expressed.

The logic of each $\mathrm{E}_{x}$ is that of a (relativised) classical modal system of type ET in the Chellas classification, i.e. the smallest system containing $P L$, closed under the rule E.RE:

$$
\text { E.RE } \frac{A \leftrightarrow B}{\mathrm{E}_{x} A \leftrightarrow \mathrm{E}_{x} B}
$$

and containing the axiom schema

$$
\text { E.T } \quad \mathrm{E}_{x} A \rightarrow A
$$

The schema E.T indicates that this is a notion of successful action. It does not matter, for the purposes of this paper, whether $x$ brings about $A$ intentionally or unintentionally, knowingly or unknowingly.

The $\mathrm{E}_{x}$ notation is from [Pörn 1977]. For the purposes of this paper, however, the (relativised) operators $\mathrm{E}_{x}$ should be regarded as standing for one of a range of possible action modalities rather than any one of them specifically. For a discussion of some candidates and their relative merits see e.g. [Chellas 1969; Pörn 1970; 1974; 1977; 1989; Åqvist 1974; Segerberg 1985; 1989; 1992; Belnap and Perloff 1988; 1992; Perloff 1991; Horty and Belnap 1995; Elgesem 1992; Hilpinen 1997]. It is likely that a comprehensive theory of rights and/or organisations would require several different notions of action and agency. In [Santos and Carmo 1996; Santos et al. 1997], for instance, it is suggested that distinguishing between direct and indirect action may be important for describing certain organisational structures. Nothing in the present paper depends on such detailed choices. As in the Kanger-Lindahl framework, the only properties assumed for the action modalities $\mathrm{E}_{x}$ are validity of the schema E.T and closure under logical equivalence, E.RE.

We conclude this introductory discussion with some brief examples to illustrate the expressive power of the language and to motivate the formal development to be undertaken in the remainder of the paper. These examples are intended to be 
simple and familiar.

Example 2.1 (Library book). Let $b$ name a borrower in a library who has some book out on loan. Let $R$ represent that this book is returned to the library by the date due. $b$ has an obligation to return the book by date due. In the Kanger framework this obligation can be represented by the following expression.

$$
\mathrm{O} \mathrm{E}_{b} R
$$

Expression (1) is not the only, nor perhaps even an adequate, representation of what we ordinarily mean by saying that $b$ has an obligation to return the book. It employs what some authors refer to as the Meinong-Chisholm analysis, whereby ' $x$ ought to bring it about that $F$ ' is taken to mean 'it ought to be that $x$ brings it about that $F^{\prime}$. It is possible to question whether these expressions are in fact equivalent. See e.g. the discussions in [Horty 1996b; 1996a; 2000; Sergot and Richards 2000; Brown 2000]. There are also some senses of 'obligation' - as when we say e.g. ' $x$ is responsible for, or held accountable for, ensuring that $F$ is the case' - which are not adequately represented by this construction. Possible formalisations of these other senses will not be discussed in this paper.

Studies of duty and right, such as Hohfeld's, usually adopt a relational perspective: the focus is on relationships between pairs of agents. So, given the truth of e.g. $\mathrm{O} \mathrm{E}_{b} R$, one is led to ask about the obligations and permissions of other agents, $a$ say, with respect to the returning of the book. One can see that, according to the logics employed, the following three possibilities are all consistent with $\mathrm{O}_{b} R$ :

(1) $a$ is obliged ${ }^{1}$ to return the book: $\mathrm{O} \mathrm{E}_{a} R$;

(2) $a$ is permitted but not obliged to return the book:

$$
\left(\mathrm{P} \mathrm{E}_{a} R \wedge \neg \mathrm{O} \mathrm{E}_{a} R\right)=\left(\mathrm{P} \mathrm{E}_{a} R \wedge \mathrm{P} \neg \mathrm{E}_{a} R\right) ;
$$

(3) $a$ is not permitted to return the book: $\neg \mathrm{P} \mathrm{E}_{a} R$.

Are there any other possibilities? It is the systematic exploration of all such possible relations that motivates the construction of the Kanger-Lindahl theories.

Notice that the three possibilities above may be distinguished by asking in turn whether $\mathrm{P}_{a} R$ is true, and if so, whether $\mathrm{P} \neg \mathrm{E}_{a} R$ is true. This is the kind of analysis that the Norman-G system described in Section 6 is designed to support.

Example 2.2 (Fence). The following example is adapted from [Lindahl 1977]. Again, no claim is made here for the completeness or adequacy of the representation. The aim is merely to illustrate some of the distinctions and nuances that can be expressed with the resources available.

Suppose $a$ and $b$ are neighbours, and let $F$ represent that there is a fence on the boundary between their adjoining properties. We want to say that $a$ has a 'right' to erect such a fence, or more generally, has a 'right' to see to it that there is such a fence. We build up a (partial) representation in stages. In the first instance it

\footnotetext{
${ }^{1}$ The expression $\mathrm{OE}_{a} R \wedge \mathrm{OE}_{b} R$ is not inconsistent. In the logics employed, it is logically equivalent to $\mathrm{O}\left(\mathrm{E}_{a} R \wedge \mathrm{E}_{b} R\right)$, but there is no principle in the logic of action to say that $a$ and $b$ could not act in such a way that they both see to it that $R$.
} 
seems reasonable to assert that the following is true:

$$
\mathrm{P} \mathrm{E}_{a} F \wedge \neg \mathrm{P} \mathrm{E}_{b} \neg F
$$

The second conjunct of this expression captures something of the idea that $b$ is not permitted to prevent $a$ from seeing to it that $F$. One could also add a conjunct $\neg \mathrm{P} \mathrm{E}_{b} \neg \mathrm{E}_{a} F$ to cover a different sense in which $b$ is forbidden to prevent $a$ from seeing to it that $F$. The ability to iterate action operators in this fashion is one of the main advantages of using the $\mathrm{E}_{x}$ device in the treatment of action. ' $x$ refrains from seeing to it that $F^{\prime}$ can be represented as $\mathrm{E}_{x} \neg \mathrm{E}_{x} F$, for example. We shall not study iterated act expressions in any detail in this paper, however. See [Sergot and Richards 2000] for more examples and some possible lines of development. Iterated act expressions are also the basis of the 'control' and 'influence' positions examined in [Pörn 1977].

Of course $a$ is not obliged to see to it that $F$, so also $\neg \mathrm{O} \mathrm{E}_{a} F$. Furthermore, $a$ 's permission to see to it that $F$ does not depend on $b$ 's actions, in the sense that the following is also true: $\mathrm{P}\left(\mathrm{E}_{a} F \wedge \neg \mathrm{E}_{b} F\right)$. Putting these together:

$$
\mathrm{PE}_{a} F \wedge \neg \mathrm{PE}_{b} \neg F \wedge \neg \mathrm{O} \mathrm{E}_{a} F \wedge \mathrm{P}\left(\mathrm{E}_{a} F \wedge \neg \mathrm{E}_{b} F\right)
$$

Compound expressions such as (2) arise frequently. The Norman-G system provides a range of syntactic abbreviations for expressing them concisely.

Expression (2) is an approximation to the concept of a 'vested right'. It is an approximation because as already observed there are other possible ways in which $b$ can be said to 'prevent' $a$ 's seeing to it that $F$, and because it fails to capture the idea that $a$ 's rights may already be infringed by unsuccessful attempts by $b$ to interfere with $a$ 's actions; furthermore (2) does not say what further rights and obligations are created if $b$ should so interfere.

In this example $b$ 's normative status in relation to $F$ is clearly symmetrical to $a$ 's and so we may add also:

$$
\mathrm{PE}_{b} F \wedge \neg \mathrm{PE}_{a} \neg F \wedge \neg \mathrm{O} \mathrm{E}_{b} F \wedge \mathrm{P}\left(\mathrm{E}_{b} F \wedge \neg \mathrm{E}_{a} F\right)
$$

But still there are a number of unresolved questions. Is it the case that $\mathrm{P} \neg F$, i.e., is it obligatory that there is a fence? Is it the case that $\mathrm{P}\left(\neg F \wedge \neg \mathrm{E}_{a} \neg F \wedge \neg \mathrm{E}_{b} \neg F\right)$ ? In fact, use of the Norman-G system demonstrates that, in the logics employed, (2) and (3) together imply

$$
\mathrm{P} \neg F \leftrightarrow \mathrm{P}\left(\neg F \wedge \neg \mathrm{E}_{a} \neg F \wedge \neg \mathrm{E}_{b} \neg F\right)
$$

i.e., it is obligatory there is a fence iff $\mathrm{O}\left(\neg F \rightarrow\left(\mathrm{E}_{a} \neg F \vee \mathrm{E}_{b} \neg F\right)\right)$. Is it the case that $\mathrm{P}\left(F \wedge \neg \mathrm{E}_{a} F \wedge \neg \mathrm{E}_{b} F\right)$ ? Perhaps some other agent, besides $a$ and $b$, may see to it that there is a fence between their adjoining properties?

The example is intended to demonstrate why there is a need for automated support even for the analysis of simple examples. The questions above are resolved straightforwardly, or rather their consequences can be explored easily, by means of the Norman-G system; Section 7 provides an illustration of how Norman-G can be used for this purpose.

The fence example also demonstrates that there may be an obligation on $a$ and $b$ together, without there being an obligation on either of them individually: it is possible that $\mathrm{O}\left(\mathrm{E}_{a} F \vee \mathrm{E}_{b} F\right)$ holds while both $\neg \mathrm{O} \mathrm{E}_{a} F$ and $\neg \mathrm{O} \mathrm{E}_{b} F$ also hold. 
Example 2.3 (Car park). Lee [1988] presents a rule-based language intended for specifying permitted, obligatory and forbidden actions. The example used for illustration concerns the rules governing parking in a University car park. For simplicity "assume that administrators have unrestricted parking privileges. Faculty, however, must obtain a parking permit to park on campus. Students must park off campus." Lee shows how such rules can be represented in the form of if/then rules whose antecedent ('body') is a conjunction of factual conditions ('is an administrator', 'has a parking permit', etc.) and whose consequent ('head') specifies an action (here, 'park') that can be permitted, obligatory, prohibited.

Leaving aside the details of the language, one might ask whether these primitives 'permitted', 'obligatory', 'prohibited' are enough, whether they cover all imaginable cases. Again, taking the relational perspective, one is immediately led to think in terms of interactions with other agents: other users of the car park, passers by, the gatekeepers who control access to the car park, the University who owns the car park and to whom the gatekeepers are responsible, and so on. An analysis based on the Hohfeldian scheme, for example, would already ask not whether there is a permission to park simpliciter but whether the administrator has a 'privilege' to park or whether this is in fact a 'right' (vis-à-vis, in turn, other users of the car park, the gatekeepers, the University). And likewise for other pairs of agents.

If in place of the informal Hohfeldian scheme, we employ the formal machinery offered by the Kanger-Lindahl theories or the extended scheme developed in this paper, the if/then rules of the representation language would take the form

\section{if conditions then normative-position}

where normative-position is one of some appropriately chosen class of normative positions. Lee's rule-based language can be regarded as a special case where the class of normative positions is a particularly simple one (and not all possibilities are covered). For more precision, more complex classes of normative positions should be considered. The question is how to decide which class of normative positions to consider, and then how to pick out one of the positions as the right one. As shown later, the number of possibilities is huge. Can the analysis be supported?

One might ask why anyone would be interested in representing the rules of a library or the rules of a car park at this level of precision. One answer is to say that a precise specification may be essential if we were assigned the task of constructing a system that advises the employees and users of a library about their duties and rights. Or if we were given the task of designing an automated system for controlling access to a car park. But really the point is this: these examples are representatives of a wide range of similar problems that arise frequently in other guises. Instead of controlling who may put cars in a car park, for instance, imagine that the car park is a computer file of some kind, and that $p(x)$ represents not that car $x$ is parked but that data entry $x$ is stored in the file. The task is to specify which agents (computer agents or human) are to be permitted to insert and delete data entries in this file. Suppose, let us say, that at midnight every night a set of electronic transactions will be generated automatically according to the data entries present in the file at that time. This is not at all a fanciful suggestion: there are many systems that operate in exactly this way. In such cases, it will be essential to specify with precision which agents are permitted to enter which data entries into which 
files and in which combinations. A gatekeeper agent $g$ who controls access to a car park is not so different from the 'file monitor' (human or computer agent) which controls access to a file. And likewise for many of the other forms of interactions that take place in regulated human and electronic societies.

\section{THE KANGER-LINDAHL THEORY}

The focus in the Kanger-Lindahl theory is on mapping out the space of logically possible legal/normative relations of given forms that can hold between pairs of agents. In order to examine the possibilities systematically, Kanger considers first what he called the 'simple types of rights relations' of two agents $a$ and $b$ with respect to some state of affairs $F$. They are represented by the expressions falling under the scheme:

$$
\pm \mathrm{O} \pm\left(\begin{array}{c}
\mathrm{E}_{a} \\
\mathrm{E}_{b}
\end{array}\right) \pm F
$$

The notation was suggested by Makinson [1986]. \pm stands for the two possibilities of affirmation and negation; the choice-scheme $\left(\begin{array}{c}\mathrm{E}_{a} \\ \mathrm{E}_{b}\end{array}\right)$ indicates the (here, two) alternatives $\mathrm{E}_{a}$ and $\mathrm{E}_{b}$. There are thus sixteen expressions falling under the scheme (4), ranging from $\mathrm{OE}_{a} F$ to $\neg \mathrm{O} \neg \mathrm{E}_{b} \neg F$. In this paper, the choice-scheme notation is used as shorthand for a set of expressions, and is mixed freely with standard set notation.

Of more interest than the 'simple types' are the various compounds that may be formed from them, or what Kanger called the 'atomic types of rights relation'. We shall build upon an observation due to Makinson [1986], that Kanger's 'atomic types', for two agents $a, b$ with respect to the bringing about of some state of affairs $F$, can be characterised as the expressions belonging to the set:

$$
\llbracket \pm \mathrm{O} \pm\left(\begin{array}{c}
\mathrm{E}_{a} \\
\mathrm{E}_{b}
\end{array}\right) \pm F \rrbracket
$$

The brackets denote maxi-conjunctions: where $\Phi$ is a choice-scheme (or set of sentences) $\llbracket \Phi \rrbracket$ stands for the set of maxi-conjunctions of $\Phi$ - the maximal consistent conjunctions of expressions belonging to $\Phi$. 'Consistent' refers to some underlying logic, here the specific logics for $\mathrm{O}$ and $\mathrm{E}_{x}$ employed by Kanger and Lindahl. 'Conjunction' means a conjunction without repetitions, and with some standard order and association of conjuncts. A conjunction is 'maximal consistent' when addition of any other conjunct from $\Phi$ yields an inconsistent conjunction: in other words, a conjunction $\Gamma$ is a maxi-conjunction of $\Phi$ if and only if $\Gamma$ is consistent, and every expression of $\Phi$ either appears as a conjunct in $\Gamma$ or is inconsistent with $\Gamma$. Note that maxi-conjunctions may contain logical redundancies (one or more conjuncts may be logically implied by the others). We shall occasionally abuse the notation and write also $\llbracket \Phi \rrbracket$ for the set of conjunctions obtained by removing all logical redundancies from the maxi-conjunctions of $\Phi$. A justification for this practice will be provided in later sections.

As can readily be checked, and will be shown more generally later (Theorem 3.1), Kanger's 'atomic types' (5) can be written as conjunctions of two simpler expres- 
sions:

$$
\llbracket \pm \mathrm{O} \pm\left(\begin{array}{c}
\mathrm{E}_{a} \\
\mathrm{E}_{b}
\end{array}\right) \pm F \rrbracket=\llbracket \pm \mathrm{O} \pm \mathrm{E}_{a} \pm F \rrbracket \cdot \llbracket \pm \mathrm{O} \pm \mathrm{E}_{b} \pm F \rrbracket
$$

Here the notation is as follows: when $\mathbf{P}$ and $\mathbf{Q}$ represent sets of expressions, $\mathbf{P} \cdot \mathbf{Q}$ stands for the set of all the consistent conjunctions that can be formed by conjoining an expression from set $\mathbf{P}$ with an expression from set $\mathbf{Q}$. (For technical reasons, it is convenient to take $\mathbf{P} \cdot \emptyset \stackrel{\text { def }}{=} \emptyset \cdot \mathbf{P} \stackrel{\text { def }}{=} \mathbf{P}$.) In more complicated expressions, in order to reduce the need for parentheses, we adopt the convention that the $\cdot$ binds more tightly than other operators. So, for example, the choice-scheme expression $\left( \pm \mathrm{O} \pm \Phi_{1} \cdot \Phi_{2}\right)$ is to be read as $\left( \pm \mathrm{O} \pm\left(\Phi_{1} \cdot \Phi_{2}\right)\right)$.

The maxi-conjunctions in

$$
\llbracket \pm \mathrm{O} \pm \mathrm{E}_{a} \pm F \rrbracket
$$

are, in the terminology of [Jones and Sergot 1992; 1993], Kanger's normative oneagent act positions. According to the logic employed by Kanger, there are six elements in (7). Following the numbering at [Lindahl 1977, p100] and eliminating logical redundancies, they are:

$$
\begin{array}{ll}
\left(\mathrm{K}_{1}\right) & \mathrm{P} \mathrm{E}_{a} F \wedge \mathrm{P} \mathrm{E}_{a} \neg F \\
\left(\mathrm{~K}_{2}\right) & \mathrm{O} \neg \mathrm{E}_{a} F \wedge \mathrm{O} \neg \mathrm{E}_{a} \neg F \\
\left(\mathrm{~K}_{3}\right) & \mathrm{O} \mathrm{E}_{a} F \\
\left(\mathrm{~K}_{4}\right) & \mathrm{P} \mathrm{E}_{a} F \wedge \mathrm{P} \neg \mathrm{E}_{a} F \wedge \mathrm{O} \neg \mathrm{E}_{a} \neg F \\
\left(\mathrm{~K}_{5}\right) & \mathrm{O} \mathrm{E}_{a} \neg F \\
\left(\mathrm{~K}_{6}\right) & \mathrm{O} \neg \mathrm{E}_{a} F \wedge \mathrm{P} \mathrm{E}_{a} \neg F \wedge \mathrm{P} \neg \mathrm{E}_{a} \neg F
\end{array}
$$

It can be seen that, by construction, these six expressions are consistent, mutually exclusive, and their disjunction is a tautology. In any given situation precisely one of them must be true, according to the logical principles employed.

For Kanger's 'atomic types' for two agents (6) there are thus $6 \times 6=36$ conjunctions to consider, of which 10 turn out to be logically inconsistent. On Kanger's analysis, therefore, there are 26 atomic types of right (for two agents with respect to the bringing about of some given state of affairs). Again, these 26 'atomic types' are internally consistent, mutually exclusive, and their disjunction is a tautology. In any given situation precisely one of them must be true, according to the logics employed. It is in this sense that Kanger can be said to provide a complete and exhaustive analysis of all the logically possible normative positions.

In general, all maxi-conjunctions of the form $\llbracket \pm \Phi \rrbracket$ have this property of exhaustiveness. Moreover, all (consistent) boolean compounds of expressions in $\Phi$ are logically equivalent to a (non-empty) disjunction of elements from $\llbracket \pm \Phi \rrbracket$. As observed by Makinson [1986], the maxi-conjunctions can be given an algebraic interpretation (as atoms of a Boolean algebra). For certain logics (those of type $E M C P$, though not for weaker ones), they give the constituents of a distributive normal form in the underlying modal logics. (They are not quite yet a normal form: for that we would need to consider not just the sentences of $\Phi$ but also all of their subsentences.) These remarks will be developed later in the paper.

The value of Makinson's suggestion, besides the conciseness of the notation, is that the characterisation of positions in terms of maxi-conjunctions emphasises 
their character rather than the specific procedures by which they happen to be generated. There are many different ways of generating the same set of maxiconjunctions. The following elementary property of maxi-conjunctions is the basis for a whole family of such procedures, and is used extensively in this paper.

THEOREM 3.1. For any choice scheme $\Phi=\Phi_{1} \cup \Phi_{2}$ ( $\Phi_{1}$ and $\Phi_{2}$ not necessarily distinct):

(1) $\llbracket \Phi_{1} \rrbracket \cdot \llbracket \Phi_{2} \rrbracket \subseteq \llbracket \Phi \rrbracket$

(2) $\llbracket \pm \Phi \rrbracket=\llbracket \pm \Phi_{1} \rrbracket \cdot \llbracket \pm \Phi_{2} \rrbracket$

Proof. (Sketch) The degenerate cases where $\llbracket \pm \Phi_{1} \rrbracket$ and/or $\llbracket \pm \Phi_{2} \rrbracket$ are empty are immediate. For the general case of part (1), it is straightforward to check that every element of $\llbracket \Phi_{1} \rrbracket \cdot \llbracket \Phi_{2} \rrbracket$ must be an element of $\llbracket \Phi \rrbracket$. For part (2), it remains to show that $\llbracket \pm \Phi \rrbracket \subseteq \llbracket \pm \Phi_{1} \rrbracket \cdot \llbracket \pm \Phi_{2} \rrbracket$. Every element of $\llbracket \pm \Phi \rrbracket$ can be written in the form $A_{1} \wedge \ldots \wedge A_{k} \wedge B_{1} \wedge \ldots \wedge B_{m} \wedge C_{1} \wedge \ldots \wedge C_{n}(k, m, n$ possibly 0$)$ where $A_{1}, \ldots, A_{k}$ belong to $\pm \Phi_{1}$ but not $\pm \Phi_{2}, C_{1}, \ldots, C_{n}$ belong to $\pm \Phi_{2}$ but not $\pm \Phi_{1}$, and $B_{1}, \ldots, B_{m}$ belong to both $\pm \Phi_{1}$ and $\pm \Phi_{2}$. Now one can show that, if $\llbracket \pm \Phi_{1} \rrbracket$ and $\llbracket \pm \Phi_{2} \rrbracket$ are not empty, $k+m>0$ and $m+n>0$. And then it is easy to check that $A_{1} \wedge \ldots \wedge A_{k} \wedge B_{1} \wedge \ldots \wedge B_{m}$ is an element of $\llbracket \pm \Phi_{1} \rrbracket$ and $B_{1} \wedge \ldots \wedge B_{m} \wedge C_{1} \wedge \ldots \wedge C_{n}$ is an element of $\llbracket \pm \Phi_{2} \rrbracket$.

Computationally: to generate the set of maxi-conjunctions $\llbracket \pm \Phi \rrbracket$, decompose the scheme (or set of sentences) $\Phi$ into smaller, not necessarily disjoint, subsets $\Phi_{1}$ and $\Phi_{2}$ (there are many different strategies for this step); (recursively) compute the sets of maxi-conjunctions $\llbracket \pm \Phi_{1} \rrbracket$ and $\llbracket \pm \Phi_{2} \rrbracket$, possibly in parallel; form all conjunctions of expressions from these sets of maxi-conjunctions; discard those conjunctions that are logically inconsistent. The steps, especially the last two steps, may be co-routined for efficiency. It is straightforward to code any such procedure as a computer program, requiring only an implementation of the inconsistency check for the generated conjunctions. Although this is not difficult-it is only fragments of the underlying modal logics that are required - it is not particularly useful either. In Section 5 we show how a little additional manipulation eliminates the need for theorem-proving techniques altogether, at least for the most common types of modal logic.

The method used to generate classes of normative positions in [Jones and Sergot 1992; 1993] is a special case of Theorem 3.1. For illustration, its generation of what are there called the 'normative fact positions' may be presented as follows:

$$
\begin{aligned}
\llbracket \pm \mathrm{O} \pm F \rrbracket & =\llbracket \pm \mathrm{O} F \rrbracket \cdot \llbracket \pm \mathrm{O} \neg F \rrbracket=\left(\begin{array}{c}
\mathrm{O} F \\
\neg \mathrm{O} F
\end{array}\right) \cdot\left(\begin{array}{c}
\mathrm{O} \neg F \\
\neg \mathrm{O} \neg F
\end{array}\right) \\
& =\left(\begin{array}{c}
\mathrm{O} F \\
\mathrm{O} \neg F \\
\mathrm{P} F \wedge \mathrm{P} \neg F
\end{array}\right) \quad \text { (with logical redundancies removed) }
\end{aligned}
$$

Equation (6) expressing Kanger's two-agent atomic types as conjunctions of oneagent types is also a special case of Theorem 3.1.

Lindahl [1977] presents a refinement and further development of Kanger's analysis. The second part of [Lindahl 1977] deals also with aspects of 'change' of normative positions. This part of Lindahl's account will not be pursued here. 
Lindahl constructs his analysis on the following set of normative one-agent act positions:

$$
\llbracket \pm \mathrm{P} \llbracket \pm \mathrm{E}_{a} \pm F \rrbracket \rrbracket
$$

where now there is a maxi-conjunction expression within the scope of the $\mathrm{P}$ operator. In words, (8) is the set of maxi-conjunction expressions of the form $\pm \mathrm{P} A$, where each $A$ is itself a maxi-conjunction of sentences of the form $\pm \mathrm{E}_{a} \pm F$. The iterated bracket notation is again from [Makinson 1986].

There are three act positions in the set

$$
\llbracket \pm \mathrm{E}_{a} \pm F \rrbracket
$$

They are:

$$
\begin{array}{ll}
\left(\mathrm{A}_{1}\right) & \mathrm{E}_{a} F \\
\left(\mathrm{~A}_{2}\right) & \mathrm{E}_{a} \neg F \\
\left(\mathrm{~A}_{3}\right) & \neg \mathrm{E}_{a} F \wedge \neg \mathrm{E}_{a} \neg F
\end{array}
$$

The third of these $\left(\mathrm{A}_{3}\right)$ is the possibility missed by Kanger's analysis. It represents a kind of 'passivity' of agent $a$ with respect to state of affairs $F$. Following Lindahl, it is convenient to define the following abbreviation:

$$
\operatorname{Pass}_{a} F \stackrel{\text { def }}{=} \neg \mathrm{E}_{a} F \wedge \neg \mathrm{E}_{a} \neg F
$$

There are $2^{3}-1=7$ expressions in the set (8). They are, numbered as in [Lindahl 1977] and with logical redundancies removed:

$$
\begin{aligned}
& \left(\mathrm{T}_{1}\right) \quad \mathrm{PE}_{a} F \wedge \mathrm{PE}_{a} \neg F \wedge \mathrm{P} \mathrm{Pass}_{a} F \\
& \left(\mathrm{~T}_{2}\right) \quad \mathrm{PE}_{a} F \wedge \mathrm{O} \neg \mathrm{E}_{a} \neg F \wedge \mathrm{P} \mathrm{Pass}_{a} F \\
& \left(\mathrm{~T}_{3}\right) \quad \mathrm{PE}_{a} F \wedge \mathrm{PE}_{a} \neg F \wedge \neg \mathrm{P} \mathrm{Pass}_{a} F \\
& \left(\mathrm{~T}_{4}\right) \quad \mathrm{O} \neg \mathrm{E}_{a} F \wedge \mathrm{PE}_{a} \neg F \wedge \mathrm{P}^{\mathrm{Pass}_{a}} F \\
& \left(\mathrm{~T}_{5}\right) \quad \mathrm{OE}_{a} F \\
& \left(\mathrm{~T}_{6}\right) \quad \mathrm{O} \mathrm{Pass}_{a} F \\
& \left(\mathrm{~T}_{7}\right) \quad \mathrm{OE}_{a} \neg F
\end{aligned}
$$

In place of Kanger's two-agent types (6), Lindahl has the following set of positions:

$$
\llbracket \pm \mathrm{P} \llbracket \pm \mathrm{E}_{a} \pm F \rrbracket \rrbracket \cdot \llbracket \pm \mathrm{P} \llbracket \pm \mathrm{E}_{b} \pm F \rrbracket \rrbracket
$$

There are $7 \times 7=49$ conjunctions to consider, of which 35 are internally consistent. These are Lindahl's 'individualistic' normative two-agent act positions. The significance of 'individualistic' will be explained in a moment.

Lindahl's construction gives a finer-grained analysis than Kanger's. For the oneagent types, five of the six in Kanger's (7) are logically equivalent to five of the seven in Lindahl's (8), as summarized in Table I.

On Lindahl's analysis, therefore, Kanger's type $\left(\mathrm{K}_{1}\right)$ can be decomposed:

$$
\left(\mathrm{K}_{1}\right) \quad \mathrm{P} \mathrm{E}_{a} F \wedge \mathrm{PE}_{a} \neg F
$$

is logically equivalent to a disjunction of two of Lindahl's types, viz.

$$
\begin{aligned}
& \left(\mathrm{T}_{1}\right) \quad \mathrm{PE}_{a} F \wedge \mathrm{PE}_{a} \neg F \wedge \mathrm{P} \mathrm{Pass}_{a} F \\
& \left(\mathrm{~T}_{3}\right) \quad \mathrm{PE} \mathrm{E}_{a} F \wedge \mathrm{PE}_{a} \neg F \wedge \neg \mathrm{P} \mathrm{Pass}_{a} F
\end{aligned}
$$


Table I

\begin{tabular}{ccccc}
\hline $\mathrm{K}_{1}$ & is logically equivalent to & $\left(\mathrm{T}_{1} \vee \mathrm{T}_{3}\right)$ \\
$\mathrm{K}_{2}$ & $\ldots$ & $\ldots$ & $\ldots$ & $\mathrm{T}_{6}$ \\
$\mathrm{~K}_{3}$ & $\ldots$ & $\ldots$ & $\ldots$ & $\mathrm{T}_{5}$ \\
$\mathrm{~K}_{4}$ & $\ldots$ & $\ldots$ & $\ldots$ & $\mathrm{T}_{2}$ \\
$\mathrm{~K}_{5}$ & $\ldots$ & $\ldots$ & $\ldots$ & $\mathrm{T}_{7}$ \\
$\mathrm{~K}_{6}$ & $\ldots$ & $\ldots$ & $\ldots$ & $\mathrm{T}_{4}$ \\
\hline
\end{tabular}

For an example of $\left(\mathrm{T}_{3}\right)$, consider a judge $(a)$ who is permitted to see to it that the prisoner is imprisoned $(F)$ and permitted to see to it that the prisoner is not imprisoned $(\neg F)$; but $a$ is not permitted to do neither of these: $\neg \mathrm{P} \mathrm{Pass}_{a} F$.

Similarly, it is possible to find equivalences between Kanger's 26 two-agent 'atomic types' (5) and disjunctions of Lindahl's corresponding 35 types (10). We omit the details: the next section presents a general result and a computational method to perform this kind of calculation.

One might wonder where the extra precision of Lindahl's analysis comes from. Notice that, since $\mathrm{P}$ is the dual of $\mathrm{O}$, Kanger's one-agent positions (7) may be written equivalently as $\llbracket \pm \mathrm{P} \pm \mathrm{E}_{a} \pm F \rrbracket$. The expression within the maxi-conjunction brackets may be seen in two ways: either as a scheme of four (not mutually exclusive) act positions $\pm \mathrm{E}_{a} \pm F$ prefixed by $\pm \mathrm{P}$, or as two mutually exclusive act positions $\mathrm{E}_{a} \pm F$ prefixed by $\pm \mathrm{P} \pm$. What is obtained by combining the second view, $\pm \mathrm{P} \pm$, with the three mutually exclusive act positions $\llbracket \pm \mathrm{E}_{a} \pm F \rrbracket$ used by Lindahl? The account of normative positions given in [Jones and Sergot 1992; 1993] uses the following, more elaborate form, in place of Lindahl's one-agent types (8):

$$
\llbracket \pm \mathrm{P} \pm \llbracket \pm \mathrm{E}_{a} \pm F \rrbracket \rrbracket=\llbracket \pm \mathrm{O} \pm \llbracket \pm \mathrm{E}_{a} \pm F \rrbracket \rrbracket
$$

(The equality here is because $\mathrm{P}$ and $\mathrm{O}$ are duals.) It turns out, however, that for the logics employed by Kanger and Lindahl the positions in set (11) are exactly the same seven as those in Lindahl's simpler form (8). By Theorem 3.1 the following holds irrespective of the logic of $\mathrm{O}$

$$
\llbracket \pm \mathrm{O} \pm \llbracket \pm \mathrm{E}_{a} \pm F \rrbracket \rrbracket=\llbracket \pm \mathrm{P} \llbracket \pm \mathrm{E}_{a} \pm F \rrbracket \rrbracket \cdot \llbracket \pm \mathrm{O} \llbracket \pm \mathrm{E}_{a} \pm F \rrbracket \rrbracket
$$

But when the logic of $\mathrm{O}$ is of type EMCP (or stronger), then also (as shown later in Section 5, Theorem 5.1):

$$
\llbracket \pm \mathrm{O} \pm \llbracket \pm \mathrm{E}_{a} \pm F \rrbracket \rrbracket=\llbracket \pm \mathrm{P} \llbracket \pm \mathrm{E}_{a} \pm F \rrbracket \rrbracket
$$

For weaker logics the equality (13) does not hold. In that case the Jones-Sergot form (11) gives a more meaningful analysis than Lindahl's (8).

There is another important respect in which Lindahl extends Kanger's analysis of two-agent 'atomic types'. In [Lindahl 1977, Ch.5] the account is extended to what are called 'collectivistic two-agent types', to cover the case where, for instance, there is an obligation on two agents which does not apply to either of them individually:

$$
\mathrm{O}\left(\mathrm{E}_{a} F \vee \mathrm{E}_{b} F\right) \wedge \neg \mathrm{OE}_{a} F \wedge \neg \mathrm{O} \mathrm{E}_{b} F
$$

Lindahl is there addressing the co-ordination of $a$ and $b$ 's actions, which introduces distinctions that cannot be expressed by conjunctions of the 'individualistic' 
types (10). The reason is simply that, in the logics employed, $\mathrm{P}$ does not distribute over conjunction (nor $\mathrm{O}$ over disjunction): $(\mathrm{P} A \wedge \mathrm{P} B) \rightarrow \mathrm{P}(A \wedge B)$ is not valid. For instance, $\mathrm{P} \mathrm{E}_{a} F \wedge \mathrm{P} \mathrm{E}_{b} F$ is consistent with both $\mathrm{P}\left(\mathrm{E}_{a} F \wedge \mathrm{E}_{b} F\right)$ and $\neg \mathrm{P}\left(\mathrm{E}_{a} F \wedge \mathrm{E}_{b} F\right)$.

Lindahl's 'collectivistic' two-agent positions are obtained by the following construction:

$$
\llbracket \pm \mathrm{P} \llbracket \pm\left(\begin{array}{c}
\mathrm{E}_{a} \\
\mathrm{E}_{b}
\end{array}\right) \pm F \rrbracket \rrbracket=\llbracket \pm \mathrm{P} \llbracket \pm \mathrm{E}_{a} \pm F \rrbracket \cdot \llbracket \pm \mathrm{E}_{b} \pm F \rrbracket \rrbracket
$$

In the EMCP-equivalent Jones-Sergot form the positions are:

$$
\llbracket \pm \mathrm{O} \pm \llbracket \pm\left(\begin{array}{c}
\mathrm{E}_{a} \\
\mathrm{E}_{b}
\end{array}\right) \pm F \rrbracket \rrbracket=\llbracket \pm \mathrm{O} \pm \llbracket \pm \mathrm{E}_{a} \pm F \rrbracket \cdot \llbracket \pm \mathrm{E}_{b} \pm F \rrbracket \rrbracket
$$

For the logics employed by Kanger and Lindahl, there are $2^{7}-1=127^{\text {'collectivis- }}$ tic normative two-agent act positions' in the sets (14) and (15). Each collectivistic type implies one of the 'individualistic' types (10); each of the 'individualistic' types is logically equivalent to a disjunction of one or more of the collectivistic types. This can be seen by reference to the table compiled by Lindahl [1977, p180], or, as shown in later sections, from a general property of maxi-conjunctions which holds when the logic of $\mathrm{O}$ is of type EMCP.

We are led now to consider the following questions:

(1) How can this account be generalised to the case of $n$ agents? This is a possibility mentioned by Lindahl but not developed by him, presumably because of the size and number of the symbolic expressions to be manipulated.

(2) How can the account be generalised to deal with related states of affairs, in the same kind of way that the 'collectivistic' positions generalise the 'individualistic'? Consider two neighbours, $a$ and $b$. Let $F$ represent that there is a fence at the front of their adjoining properties, and $G$ that there is a fence at the back of their properties. Suppose both neighbours are permitted to see to it that there is a fence at the front, $\mathrm{P} \mathrm{E}_{a} F \wedge \mathrm{PE}_{b} F$, and permitted to see to it that there is a fence at the back, $\mathrm{P} \mathrm{E}_{a} G \wedge \mathrm{P} \mathrm{E}_{b} G$. We might nevertheless want to distinguish between the case represented by $\mathrm{P}\left(\mathrm{E}_{a} F \wedge \mathrm{E}_{a} G\right) \wedge \mathrm{P}\left(\mathrm{E}_{b} F \wedge \mathrm{E}_{b} G\right)$ and the case represented by $\neg \mathrm{P}\left(\mathrm{E}_{a} F \wedge \mathrm{E}_{a} G\right) \wedge \neg \mathrm{P}\left(\mathrm{E}_{b} F \wedge \mathrm{E}_{b} G\right)$. It is conceivable that there could be other constraints, such as that represented by $\mathrm{O}\left(\mathrm{E}_{a} F \leftrightarrow \mathrm{E}_{a} G\right)$, i.e. $\neg \mathrm{P}\left(\mathrm{E}_{a} F \wedge \neg \mathrm{E}_{a} G\right) \wedge$ $\neg \mathrm{P}\left(\mathrm{E}_{a} G \wedge \neg \mathrm{E}_{a} F\right)$.

(3) To what extent can these various constructions be generalised to other, weaker logics than those employed by Kanger and Lindahl? Which features of the theory are properties of the specific logics employed, and which of maxi-conjunctions in general?

(4) Lindahl's construction yields a finer-grained analysis than Kanger's. Is there similarly a finer-grained analysis than Lindahl's? Is there a finest analysis?

The first three questions are answered in Sections 4 and 5. The last can be answered as follows. For one agent $a$ and one state of affairs $F$, Lindahl bases his analysis on the set of three act positions $\llbracket \pm \mathrm{E}_{a} \pm F \rrbracket$. A finer analysis is obtained by taking instead the act positions from the following scheme:

$$
\llbracket \pm \mathrm{E}_{a} \pm F \rrbracket \cdot \llbracket \pm F \rrbracket
$$


Table II

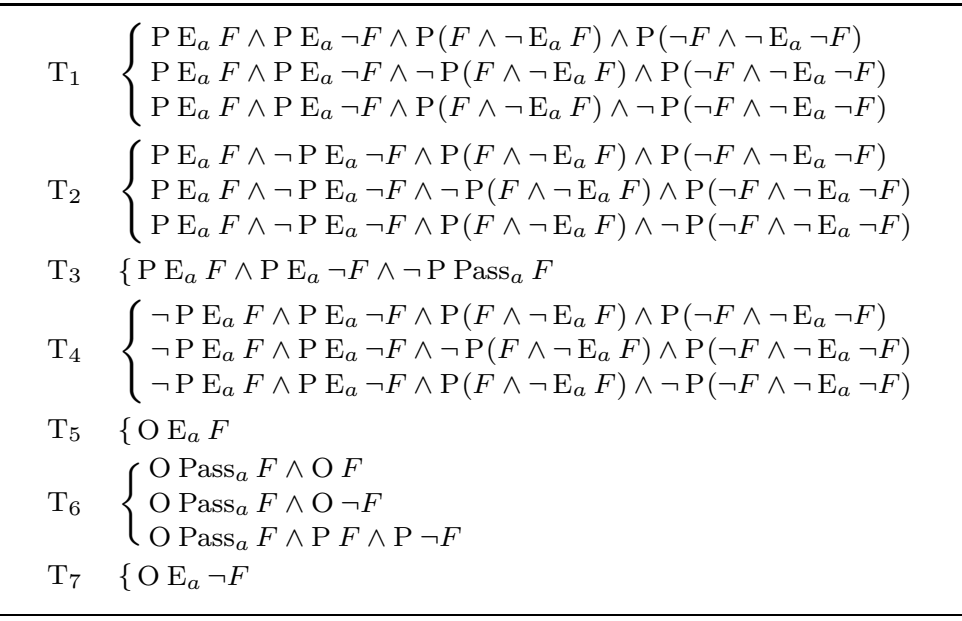

We might call these 'cumulative fact/act positions'. There are four such positions:

$\left(\mathrm{A}_{1}\right) \quad \mathrm{E}_{a} F$

$\left(\mathrm{A}_{2}\right) \quad \mathrm{E}_{a} \neg F$

$\left(\mathrm{A}_{3 a}\right) \quad F \wedge \neg \mathrm{E}_{a} F \quad$ (which is logically equivalent to $\operatorname{Pass}_{a} F \wedge F$ )

$\left(\mathrm{A}_{3 b}\right) \quad \neg F \wedge \neg \mathrm{E}_{a} \neg F \quad$ (which is logically equivalent to $\operatorname{Pass}_{a} F \wedge \neg F$ )

Lindahl's 'passive' act position $\left(A_{3}\right)$ does not distinguish between $\left(A_{3 a}\right)$ and $\left(A_{3 b}\right)$.

The corresponding 'normative act positions' are:

$$
\llbracket \pm \mathrm{O} \pm \llbracket \pm \mathrm{E}_{a} \pm F \rrbracket \cdot \llbracket \pm F \rrbracket \rrbracket
$$

There are $2^{4}-1=15$ conjunctions in the set (17), as compared with the seven $\left(\mathrm{T}_{1}\right)-\left(\mathrm{T}_{7}\right)$ constructed in Lindahl's analysis. For completeness they are listed in Table II. Three are identical to Lindahl's $\left(\mathrm{T}_{3}\right),\left(\mathrm{T}_{5}\right)$ and $\left(\mathrm{T}_{7}\right)$; the other four of Lindahl's types are each logically equivalent to a disjunction of three conjunctions from (17). Just as Lindahl is able to give examples to illustrate the ambiguity in Kanger's type $\left(\mathrm{K}_{1}\right)$, so it is easy to find examples to illustrate the ambiguities in Lindahl's types $\left(\mathrm{T}_{1}\right),\left(\mathrm{T}_{2}\right),\left(\mathrm{T}_{4}\right),\left(\mathrm{T}_{6}\right)$.

For two-agent positions, the corresponding expressions for 'individualistic' and 'collectivistic' positions are, respectively:

$$
\begin{gathered}
\llbracket \pm \mathrm{O} \pm \llbracket \pm \mathrm{E}_{a} \pm F \rrbracket \cdot \llbracket \pm F \rrbracket \rrbracket \cdot \llbracket \pm \mathrm{O} \pm \llbracket \pm \mathrm{E}_{b} \pm F \rrbracket \cdot \llbracket \pm F \rrbracket \rrbracket \\
\llbracket \pm \mathrm{O} \pm \llbracket \pm\left(\begin{array}{c}
\mathrm{E}_{a} \\
\mathrm{E}_{b}
\end{array}\right) \pm F \rrbracket \cdot \llbracket \pm F \rrbracket \rrbracket=\llbracket \pm \mathrm{O} \pm \llbracket \pm \mathrm{E}_{a} \pm F \rrbracket \cdot \llbracket \pm \mathrm{E}_{b} \pm F \rrbracket \cdot \llbracket \pm F \rrbracket \rrbracket
\end{gathered}
$$

It is convenient to introduce a special notation for cumulative fact/act positions. We use the following (for any agents $a, b, \ldots$ and formula $F$ ):

$$
\left\langle \pm \mathrm{E}_{a} \pm F\right\rangle \stackrel{\text { def }}{=} \llbracket \pm \mathrm{E}_{a} \pm F \rrbracket \cdot \llbracket \pm F \rrbracket
$$

ACM Transactions on Computational Logic, Vol. 2, No. 4, October 2001. 


$$
\left\langle \pm\left(\begin{array}{c}
\mathrm{E}_{a} \\
\mathrm{E}_{b} \\
\vdots
\end{array}\right) \pm F\right\rangle \stackrel{\text { def }}{=} \llbracket \pm\left(\begin{array}{c}
\mathrm{E}_{a} \\
\mathrm{E}_{b} \\
\vdots
\end{array}\right) \pm F \rrbracket \cdot \llbracket \pm F \rrbracket
$$

With this notation, expressions (18) and (19) are written

$$
\llbracket \pm \mathrm{O} \pm\left\langle \pm \mathrm{E}_{a} \pm F\right\rangle \rrbracket \cdot \llbracket \pm \mathrm{O} \pm\left\langle \pm \mathrm{E}_{b} \pm F\right\rangle \rrbracket
$$

and

$$
\llbracket \pm \mathrm{O} \pm\left\langle \pm\left(\begin{array}{c}
\mathrm{E}_{a} \\
\mathrm{E}_{b}
\end{array}\right) \pm F\right\rangle \rrbracket=\llbracket \pm \mathrm{O} \pm\left\langle \pm \mathrm{E}_{a} \pm F\right\rangle \cdot\left\langle \pm \mathrm{E}_{b} \pm F\right\rangle \rrbracket
$$

respectively.

When the logic of $\mathrm{O}$ is of type EMCP or stronger, constructions (17) for one agent and (19) for any pair of agents are - effectively - the finest-grained set of normative positions that can be constructed for a given state of affairs, respectively. The next section explains what is meant by 'finest-grained'. An explanation of the term 'effectively', and constructions for logics weaker than type EMCP, are discussed in the section following that.

\section{PARTITIONS}

Lindahl's construction yields a finer-grained analysis than Kanger's. But Kanger's analysis is also exhaustive, in the sense that his 'atomic types' are logically consistent, mutually exclusive, and their disjunction is a tautology. Kanger's analysis and Lindahl's analysis are both exhaustive, but Lindahl's is finer than Kanger's. We now formalise these notions.

We begin by defining a syntactic version of the standard notion of a partition of a set whereby a set is partitioned into non-empty disjoint subsets. All definitions are given with respect to some underlying logic $\Lambda$. Since $\Lambda$ is usually obvious from context we write $\vdash A$ for $A \in \Lambda$. The only assumption we make in this section is that $\Lambda$ includes classical propositional logic, i.e. contains all tautologies $P L$ and is closed under modus ponens.

Definition 4.1. Let $\mathbf{P}=\left\{P_{1}, P_{2}, \ldots\right\}$ be a set of sentences and $Q$ a sentence of the language of $\Lambda$. Then $\mathbf{P}=\left\{P_{1}, P_{2}, \ldots\right\}$ is a $\Lambda$-partition of $Q$ iff it satisfies the following conditions:

(1) every element $P_{i}$ of $\mathbf{P}$ is logically consistent: $\forall \neg P_{i}$;

(2) every element $P_{i}$ of $\mathbf{P}$ logically implies $Q: \vdash P_{i} \rightarrow Q$;

(3) distinct elements of $\mathbf{P}$ are mutually exclusive: $\vdash \neg\left(P_{i} \wedge P_{j}\right) \quad(i \neq j)$;

(4) the set $\mathbf{P}$ 'exhausts' $Q: \vdash Q \rightarrow \bigvee_{P \in \mathbf{P}} P$.

Conditions (2) and (4) together are: $\vdash Q \leftrightarrow \bigvee_{P \in \mathbf{P}} P$.

When $Q$ is a tautology we shall say that $\mathbf{P}$ is a complete $\Lambda$-partition, or simply a $\Lambda$-partition. Where context permits we omit the $\Lambda$-prefix and simply say 'partition'. In what follows partitions will be finite sets.

Example 4.2. All of the following (the terminology is from [Jones and Sergot 1992; 1993]) are (complete) partitions: 
—fact positions: $\llbracket \pm F \rrbracket=\{F, \neg F\}$;

-Lindahl's one-agent act positions: $\llbracket \pm \mathrm{E}_{a} \pm F \rrbracket=\left\{\mathrm{E}_{a} F, \mathrm{E}_{a} \neg F, \operatorname{Pass}_{a} F\right\}$;

一normative fact positions: $\llbracket \pm \mathrm{O} \pm F \rrbracket=\{\mathrm{O} F, \mathrm{O} \neg F, \mathrm{P} F \wedge \mathrm{P} \neg F\}$;

-Lindahl's normative one-agent act positions $\left(\mathrm{T}_{1}\right)-\left(\mathrm{T}_{7}\right), \llbracket \pm \mathrm{P} \llbracket \pm \mathrm{E}_{a} \pm F \rrbracket \rrbracket$.

In general, any maxi-conjunction of the form $\llbracket \pm \Phi \rrbracket$ is a (complete) partition. In contrast:

- The act positions used by Kanger, $\pm \mathrm{E}_{a} \pm F$, are not mutually exclusive, whereas $\mathrm{E}_{a} \pm F=\left\{\mathrm{E}_{a} F, \mathrm{E}_{a} \neg F\right\}$ are mutually exclusive but do not form a complete partition.

Naturally, if $\left\{P_{1}, \ldots, P_{n}\right\}$ is a set of consistent, mutually exclusive sentences, then $\left\{P_{1}, \ldots, P_{n}\right\}$ is a partition of $P_{1} \vee \ldots \vee P_{n}$.

$\Lambda$-partitions are just syntactic analogues of the standard notion of a partition of a set. The two are easily related. For any model $\mathcal{M}$ of $\Lambda$, let $\|Q\|^{\mathcal{M}}$ denote the 'truth set' of $Q$, i.e. the set of possible worlds of $\mathcal{M}$ at which $Q$ is true. The exact structure of $\mathcal{M}$ does not matter. Then the set of sentences $\mathbf{P}=\left\{P_{1}, P_{2}, \ldots\right\}$ is a $\Lambda$-partition of $Q$ when, for all models $\mathcal{M}$ of $\Lambda$, the sets $\left\|P_{1}\right\|^{\mathcal{M}},\left\|P_{2}\right\|^{\mathcal{M}}, \ldots$ partition the set $\|Q\|^{\mathcal{M}}$.

In view of this observation, it would be possible to eliminate the need for Definition 4.1 altogether and use instead the set-theoretic language indicated above, identifying each sentence with the set of all maximal consistent sets that contain it, and taking the notion of partition in its ordinary set-theoretic sense. There would be some advantage in doing so. For example, as pointed out by David Makinson [personal communication] Theorem 3.1 can be generalized and given an abstract, purely set-theoretical formulation. When $X, Y$ are any collections of subsets of a fixed set $S$, write $\llbracket \pm X \rrbracket$ for the set of all elements of $X$ and their complements (w.r.t. $S$ ). Write $\llbracket X \rrbracket$ for the set of all minimal (under inclusion) non-empty intersections of elements of $X$. Write $\llbracket X \rrbracket \cdot \llbracket Y \rrbracket$ for the set of all non-empty intersections of an element of $\llbracket X \rrbracket$ with an element of $\llbracket Y \rrbracket$. Then (i) for all subsets $X$ of $S, \llbracket \pm X \rrbracket$ is a partition (in the standard set-theoretic sense) of $S$; and (ii) for all subsets $X$, $Y$ of $S, \llbracket \pm(X \cup Y) \rrbracket=\llbracket \pm X \rrbracket \cdot \llbracket \pm Y \rrbracket$. Moreover, there are well known properties of ordinary, set-theoretic partitions that could profitably be exploited.

We have chosen to stick to the syntactic variant defined in 4.1, however, because its application is more immediate in the present context. Furthermore, given a set of sentences, it is still necessary to check whether they constitute a partition, and for this purpose Definition 4.1 is more useful. We record in this section a number of properties of (syntactic) partitions that will be used later. All of them are easy to check, either directly from Definition 4.1 or by translating first to the set-theoretic analogue.

Proposition 4.3. Let $\mathbf{P}$ and $\mathbf{Q}$ be partitions of some sentence $R$. Then the set of conjunctions $\mathbf{P} \cdot \mathbf{Q}$ is non-empty and is also a partition of $R$.

In the above, $\mathbf{P} \cdot \mathbf{Q}$ must be non-empty, else $R$ is logically inconsistent and $\mathbf{P}$ and $\mathbf{Q}$ could not be partitions. We now proceed to define some relations between partitions.

ACM Transactions on Computational Logic, Vol. 2, No. 4, October 2001. 
Definition 4.4. Let $\mathbf{P}$ and $\mathbf{Q}$ be partitions of some sentence $R$. $\mathbf{P}$ and $\mathbf{Q}$ are equivalent $(\mathbf{P} \equiv \mathbf{Q})$ iff their elements are pairwise logically equivalent, i.e. iff there is a bijection $f: \mathbf{P} \rightarrow \mathbf{Q}$ such that $\vdash P \leftrightarrow f(P)$ for all elements $P$ of $\mathbf{P}$.

Now we define an ordering on partitions.

Definition 4.5. Let $\mathbf{P}$ and $\mathbf{Q}$ be partitions of some sentence $R$. $\mathbf{P}$ is a refinement of $\mathbf{Q}(\mathbf{P} \geq \mathbf{Q})$ iff every element of $\mathbf{P}$ logically implies some element of $\mathbf{Q}$ :

$$
\mathbf{P} \geq \mathbf{Q} \quad \text { iff } \quad \forall P \in \mathbf{P} \exists Q \in \mathbf{Q} \text { such that } \vdash P \rightarrow Q .
$$

When $\mathbf{P} \geq \mathbf{Q}$ we shall also say that partition $\mathbf{P}$ refines partition $\mathbf{Q}$.

Example 4.6.

- Here is an instance of a general property to be established in a moment:

$$
\llbracket \pm P \rrbracket \cdot \llbracket \pm Q \rrbracket \geq \llbracket \pm P \rrbracket
$$

—Lindahl [1977, p100] provides a table comparing his atomic (one-agent) types with those of Kanger. From the table it is clear that Lindahl's types (which are a (complete) partition) are a refinement of Kanger's:

$$
\llbracket \pm \mathrm{P} \llbracket \pm \mathrm{E}_{a} \pm F \rrbracket \rrbracket \geq \llbracket \pm \mathrm{O} \pm \mathrm{E}_{a} \pm F \rrbracket
$$

In later sections we shall be able to establish this relationship without having to compute these sets explicitly. It holds when the logic of O is of type EMCP.

- The procedure used in [Jones and Sergot 1992; 1993] constructs a set of maxiconjunctions that is a refinement of Lindahl's normative one-agent act positions:

$$
\llbracket \pm \mathrm{O} \pm \llbracket \pm \mathrm{E}_{a} \pm F \rrbracket \rrbracket \geq \llbracket \pm \mathrm{P} \llbracket \pm \mathrm{E}_{a} \pm F \rrbracket \rrbracket
$$

(This is just a corollary of Theorem 3.1. See Example 4.9 below.)

—Lindahl's 'collectivistic' two-agent types are a refinement of the 'individualistic' types:

$$
\llbracket \pm \mathrm{P} \llbracket \pm\left(\begin{array}{c}
\mathrm{E}_{a} \\
\mathrm{E}_{b}
\end{array}\right) \pm F \rrbracket \rrbracket \geq \llbracket \pm \mathrm{P} \llbracket \pm \mathrm{E}_{a} \pm F \rrbracket \rrbracket \cdot \llbracket \pm \mathrm{P} \llbracket \pm \mathrm{E}_{b} \pm F \rrbracket \rrbracket
$$

This can be seen by examination of the table compiled by Lindahl [1977, p180] but again it can be established, without evaluating the two expressions in full, by means of general properties of maxi-conjunctions. It holds when the logic of $\mathrm{O}$ is of type EMCP.

- Normative positions based on cumulative fact/act postions (17) are a refinement of Lindahl's normative one-agent act positions:

$$
\llbracket \pm \mathrm{O} \pm\left\langle \pm \mathrm{E}_{a} \pm F\right\rangle \rrbracket \geq \llbracket \pm \mathrm{O} \pm \llbracket \pm \mathrm{E}_{a} \pm F \rrbracket \rrbracket \geq \llbracket \pm \mathrm{P} \llbracket \pm \mathrm{E}_{a} \pm F \rrbracket \rrbracket
$$

This can be seen by inspection of Table II above.

- The same is true of the corresponding two agent positions:

$$
\llbracket \pm \mathrm{O} \pm\left\langle \pm\left(\begin{array}{c}
\mathrm{E}_{a} \\
\mathrm{E}_{b}
\end{array}\right) \pm F\right\rangle \rrbracket \geq \llbracket \pm \mathrm{O} \pm \llbracket \pm\left(\begin{array}{c}
\mathrm{E}_{a} \\
\mathrm{E}_{b}
\end{array}\right) \pm F \rrbracket \rrbracket \geq \llbracket \pm \mathrm{P} \llbracket \pm\left(\begin{array}{c}
\mathrm{E}_{a} \\
\mathrm{E}_{b}
\end{array}\right) \pm F \rrbracket \rrbracket
$$


Proposition 4.7. Let $\mathbf{P}, \mathbf{Q}, \mathbf{R}$ be partitions of some sentence $S$.

(1) $\mathbf{P} \equiv \mathbf{Q}$ iff $\mathbf{P} \geq \mathbf{Q}$ and $\mathbf{Q} \geq \mathbf{P}$;

(2) $\mathbf{P} \cdot \mathbf{Q} \geq \mathbf{P}$ and $\mathbf{P} \cdot \mathbf{Q} \geq \mathbf{Q}$;

(3) $\mathbf{P} \cdot \mathbf{Q} \equiv \mathbf{P}$ iff $\mathbf{P} \geq \mathbf{Q}$;

(4) Moreover, the conjunction operator - is the 'meet' operator (glb) for partitions: if $\mathbf{R} \geq \mathbf{P}$ and $\mathbf{R} \geq \mathbf{Q}$ then $\mathbf{R} \geq \mathbf{P} \cdot \mathbf{Q}$.

COROllary 4.8. For sets of sentences $\Phi_{1} \subseteq \Phi_{2}: \llbracket \pm \Phi_{2} \rrbracket \geq \llbracket \pm \Phi_{1} \rrbracket$.

Proof. Follows immediately from Theorem 3.1 and Proposition 4.7, part (2).

Example 4.9. Since $\mathrm{P}$ is the dual of $\mathrm{O}, \pm \mathrm{P} \llbracket \pm \mathrm{E}_{a} \pm F \rrbracket \subseteq \pm \mathrm{O} \pm \llbracket \pm \mathrm{E}_{a} \pm F \rrbracket$, and hence

$$
\llbracket \pm \mathrm{O} \pm \llbracket \pm \mathrm{E}_{a} \pm F \rrbracket \rrbracket \geq \llbracket \pm \mathrm{P} \llbracket \pm \mathrm{E}_{a} \pm F \rrbracket \rrbracket
$$

as observed in Example 4.6 above.

Definition 4.10. For $\mathbf{P}$ a set of sentences and $Q$ any expression:

$$
\mathbf{P} / Q \stackrel{\text { def }}{=} \quad\{P \in \mathbf{P} \mid P \wedge Q \text { consistent }\} .
$$

For example: suppose that in the analysis of some scenario or set of regulations, it is determined that $\mathrm{OE}_{a} F$ is true. The library example of Section 2 is of this form. Then

$$
\llbracket \pm \mathrm{O} \pm \llbracket \pm \mathrm{E}_{b} \pm F \rrbracket \rrbracket / \mathrm{O} \mathrm{E}_{a} F
$$

represents the (Jones-Sergot) normative one-agent act positions consistent with $\mathrm{O} \mathrm{E}_{a} F$. The 'collectivistic' two-agent act positions consistent with $\mathrm{O}_{a} F$ are given by the expression:

$$
\llbracket \pm \mathrm{O} \pm \llbracket \pm\left(\begin{array}{c}
\mathrm{E}_{a} \\
\mathrm{E}_{b}
\end{array}\right) \pm F \rrbracket \rrbracket / \mathrm{O} \mathrm{E}_{a} F
$$

We can say more about the structure of partitions $\mathbf{P}$ and $\mathbf{Q}$ in the case that $\mathbf{P}$ is a refinement of $\mathbf{Q}$. This will be exploited in the automated procedures to be presented in later sections. It is easy to check that when $\mathbf{P} \geq \mathbf{Q}$ and $Q$ is an element of $\mathbf{Q}$ then $\mathbf{P} / Q$ is also the set of elements of $\mathbf{P}$ that logically imply $Q$. Indeed, when $\mathbf{P} \geq \mathbf{Q}$ and $Q$ is an element of $\mathbf{Q}$ then $\mathbf{P} / Q$ is a $\Lambda$-partition of $Q$. And further: the set $\mathbf{P}$ itself is partitioned (standard set notion) into the collection of disjoint subsets $\mathbf{P} / Q_{i}$ where the $Q_{i}$ are the elements of $\mathbf{Q}$. The relationships are summarised in Figure 2. (The rectangles can be seen as Venn diagrams of the corresponding truth sets, moved apart to show the structure of the two partitions.)

We are now in a position to summarise the relationship between Kanger's (oneagent) 'atomic types', Lindahl's more refined version, the more complicated construction used in [Jones and Sergot 1992; 1993], and the maxi-conjunctions identified at the end of Section 3 as a further refinement still. We include for completeness the set of 'normative fact positions' $₫ \pm \mathrm{O} \pm F \rrbracket$. The Kanger and Lindahl forms are not refinements of this last one. They have a weaker relationship which we term an elaboration. 


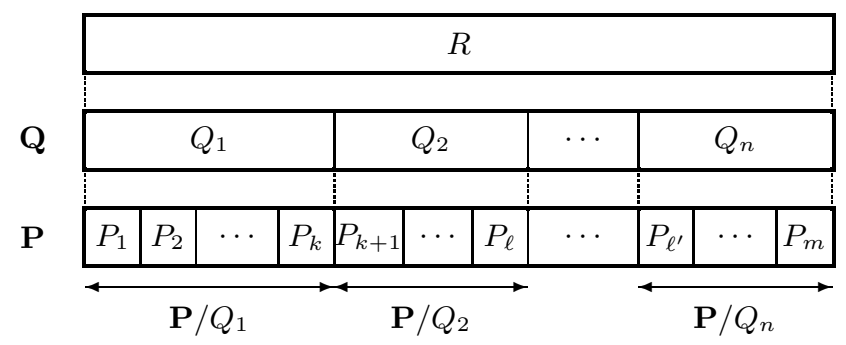

Fig. 2. Partitions $\mathbf{P}$ and $\mathbf{Q}$ of $R$ with $\mathbf{P} \geq \mathbf{Q}$

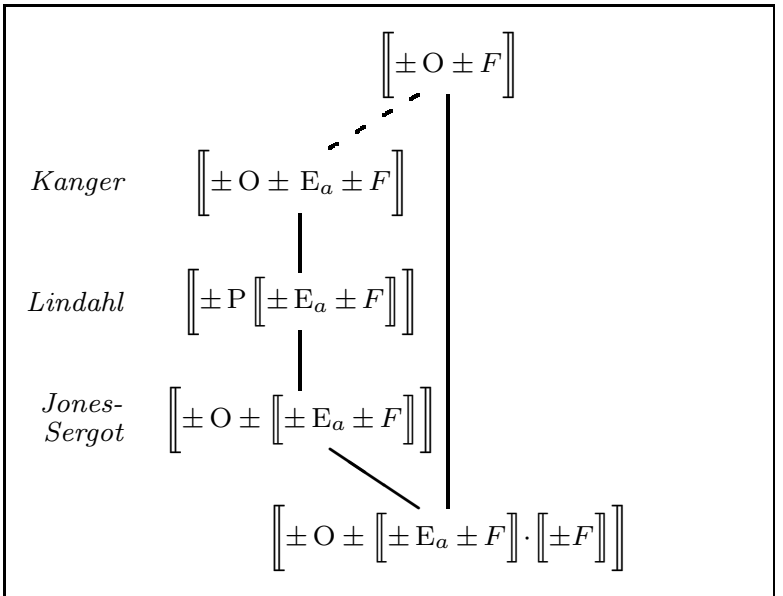

Fig. 3. Normative one-agent act positions

Definition 4.11. Let $\mathbf{P}$ and $\mathbf{Q}$ be partitions of some sentence $R$. $\mathbf{P}$ is an elaboration of $\mathbf{Q}(\mathbf{P} \succeq \mathbf{Q})$ iff for every $Q \in \mathbf{Q}$ there is a $P \in \mathbf{P}$ such that $\vdash P \rightarrow Q$.

Example 4.12. Consider the 'one-agent act positions' used by Lindahl:

$$
\llbracket \pm \mathrm{E}_{a} \pm F \rrbracket=\left\{\mathrm{E}_{a} F, \mathrm{E}_{a} \neg F, \operatorname{Pass}_{a} F\right\}
$$

Since $\mathrm{E}_{a}$ is a 'success' operator, $\llbracket \pm \mathrm{E}_{a} \pm F \rrbracket$ is an elaboration of $\llbracket \pm F \rrbracket$. But $\llbracket \pm \mathrm{E}_{a} \pm F \rrbracket$ is not a refinement of $\llbracket \pm F \rrbracket$ because $\operatorname{Pass}_{a} F=\neg \mathrm{E}_{a} F \wedge \neg \mathrm{E}_{a} \neg F$ does not imply any element of $\llbracket \pm F \rrbracket$.

It is possible to establish various relationships between refinements, elaborations and equivalences of partitions, but we shall not do so here. The relationships between the various forms of one-agent positions are summarised in Figure 3. The broken line represents an elaboration. The solid lines are refinements. The partitions at the bottom of the diagram are refinements (elaborations) of those higher up.

Finally, the following properties are useful for performing (hand) computations.

Proposition 4.13. For $\mathbf{P}$ and $\mathbf{Q}$ any sets of sentences and $R$ any expression: $\mathbf{P} \cdot \mathbf{Q} / R \subseteq(\mathbf{P} / R) \cdot(\mathbf{Q} / R)$. 
In general, Proposition (4.13) provides only an upper bound on $\mathbf{P} \cdot \mathbf{Q} / R$ and cannot be strengthened to an equality. There are some common special cases, however. The following is useful.

Proposition 4.14. Let $\mathbf{P}, \mathbf{Q}, \mathbf{R}$ be partitions of some sentence $S$ such that $\mathbf{P} \geq \mathbf{R}$. Then for any $R \in \mathbf{R}: \mathbf{P} \cdot \mathbf{Q} / R=(\mathbf{P} / R) \cdot(\mathbf{Q} / R)$.

Corollary 4.15. For any choice schemes (or sets of sentences) $\Phi_{1}$ and $\Phi_{2}$, and any sentence $A \in\left( \pm \Phi_{1} \cup \pm \Phi_{2}\right)$ :

$$
\left(\llbracket \pm \Phi_{1} \rrbracket \cdot \llbracket \pm \Phi_{2} \rrbracket\right) / A=\left(\llbracket \pm \Phi_{1} \rrbracket / A\right) \cdot\left(\llbracket \pm \Phi_{2} \rrbracket / A\right) .
$$

\section{NORMATIVE POSITIONS}

There are two main questions to consider:

(a) Given logic $\Lambda$ and scheme (set of sentences) $\Phi$, what is the set of maxiconjunctions $\llbracket \pm \Phi \rrbracket ?$

(b) For given logic $\Lambda$, which schemes (sets of sentences) $\Phi$ yield the most meaningful, or useful, sets of maxi-conjunctions $\llbracket \pm \Phi \rrbracket$ ?

\subsection{Maxi-conjunctions}

We begin by looking at a special case of question (a), focussing on maxi-conjunctions of the form:

$$
\llbracket \pm \mathrm{O} \pm \mathbf{A} \rrbracket=\llbracket \pm \mathrm{P} \pm \mathbf{A} \rrbracket \quad(\mathbf{A} \text { a complete partition })
$$

We assume only that $\mathbf{A}$ is a complete partition. We shall not take into account the structure of sentences in $\mathbf{A}$ and the possibility of rules and axiom schemas in $\Lambda$ that would allow reductions of certain iterated modalities. In this paper we restrict attention to the logics employed by Kanger and Lindahl: type EMCP for the logic of $\mathrm{O}$ and (later in the section) type ET for the action modalities $\mathrm{E}_{x}$. Elsewhere [Sergot 1996] we set out the structure of maxi-conjunctions of the form (20) for a range of logics from type $E P$ to type $E M C P$, and beyond.

For $\mathrm{O}$ of type EMCP and $\mathbf{A}$ a complete partition, the maxi-conjunctions in $\llbracket \pm \mathrm{O} \pm \mathbf{A} \rrbracket$ have a particularly simple form. In that case $\llbracket \pm \mathrm{O} \pm \mathbf{A} \rrbracket$ is (equivalent to) $\llbracket \pm \mathrm{P} \mathbf{A} \rrbracket$. When $\mathbf{A}=\left\{A_{1}, \ldots, A_{n}\right\}$, this is the set of conjunctions of the form

$$
\pm \mathrm{P} A_{1} \wedge \ldots \wedge \mathrm{P} A_{j} \wedge \ldots \wedge \pm \mathrm{P} A_{n}
$$

that is, conjunctions such that, for each $A_{i} \in \mathbf{A}$, there is a conjunct of the form $\mathrm{P} A_{i}$ or $\neg \mathrm{P} A_{i}$, and at least one conjunct is of the form $\mathrm{P} A_{j}$. We write $\pi \mathbf{A}(\pi$ possibly subscripted) to stand for any conjunction of the form (21). $\pi^{+} \mathbf{A}$ is the set of the permissible $A_{i}$ in $\pi \mathbf{A}$, i.e.

$$
\pi^{+} \mathbf{A} \stackrel{\text { def }}{=}\left\{A_{i} \in \mathbf{A} \mid \pi \mathbf{A} \vdash \mathrm{P} A_{i}\right\}
$$

$\pi^{-} \mathbf{A}$ is the set of the 'prohibited' $A_{i}$, i.e.

$$
\pi^{-} \mathbf{A} \stackrel{\text { def }}{=}\left\{A_{i} \in \mathbf{A} \mid \pi \mathbf{A} \vdash \neg \mathbf{P} A_{i}\right\}=\mathbf{A}-\pi^{+} \mathbf{A} .
$$

Theorem 5.1. Let $\mathbf{A}=\left\{A_{1}, \ldots, A_{n}\right\}$ be a complete partition. When the logic of $\mathrm{O}$ is of type EMCP the set of maxi-conjunctions:

$$
\llbracket \pm \mathrm{O} \pm \mathbf{A} \rrbracket=\llbracket \pm \mathrm{P} \pm \mathbf{A} \rrbracket
$$

ACM Transactions on Computational Logic, Vol. 2, No. 4, October 2001. 
is equivalent (Definition 4.4) to the set of conjunctions $\pi \mathbf{A}$, i.e., conjunctions $C_{1} \wedge$ $\ldots \wedge C_{n}$ where each conjunct $C_{i}$ is either $\mathrm{P} A_{i}$ or $\neg \mathrm{P} A_{i}$ and there is at least one conjunct $C_{j}$ of the form $\mathrm{P} A_{j}$.

Proof. Clearly every conjunction $\pi \mathbf{A}$ is consistent, and maximal for expressions falling under the scheme $\pm \mathrm{P} \mathbf{A}$. The conjunction $\neg \mathrm{P} A_{1} \wedge \ldots \wedge \neg \mathrm{P} A_{n}$, where there is no conjunct of the form $\mathrm{P} A_{j}$, is inconsistent:

$$
\begin{aligned}
\left(\neg \mathrm{P} A_{1} \wedge \ldots \wedge \neg \mathrm{P} A_{n}\right) & \leftrightarrow \neg\left(\mathrm{P} A_{1} \vee \ldots \vee \mathrm{P} A_{n}\right) & & (\mathrm{PL}) \\
& \leftrightarrow \neg \mathrm{P}\left(A_{1} \vee \ldots \vee A_{n}\right) & & (\mathrm{O} . \mathrm{M}, \mathrm{O} . \mathrm{C}, P L) \\
& \leftrightarrow \neg \mathrm{P} \top & & (\mathbf{A} \text { a complete partition, O.RE) } \\
& \leftrightarrow \perp & & (\mathrm{O} . \mathrm{P}, P L)
\end{aligned}
$$

The other expressions to consider are those falling under the scheme $\pm \mathrm{P} \neg \mathbf{A}$, i.e. those of the form $\pm \mathrm{P} \neg A_{j}, A_{j} \in \mathbf{A}$. It remains to show that every such expression is either inconsistent with or implied by every conjunction $\pi \mathbf{A}$. We make use of the following rule P.RM, which is available whenever the logic of $\mathrm{O}$ contains O.RE and O.M:

$$
\text { P.RM } \frac{A \rightarrow B}{\mathrm{P} A \rightarrow \mathrm{PB} B}
$$

Consider any element of the form $\mathrm{P} \neg A_{j}, A_{j} \in \mathbf{A}$. Since $\mathbf{A}$ is a partition, $\vdash A_{i} \rightarrow \neg A_{j}$ for all $i \neq j$, and so $\vdash \mathrm{P} A_{i} \rightarrow \mathrm{P} \neg A_{j}$ by P.RM. So $\mathrm{P} \neg A_{j}$ is implied by any conjunction $\pi \mathbf{A}$ containing a conjunct $\mathrm{P} A_{i}$ for any $i \neq j$. The only conjunction $\pi \mathbf{A}$ not of this form is the one for which $\pi^{+} \mathbf{A}=\left\{A_{j}\right\}$. But this $\pi \mathbf{A}$ is logically equivalent to $\mathrm{O} A_{j}$, and so is inconsistent with $\mathrm{P} \neg A_{j}=\neg \mathrm{O} A_{j}$.

Finally, consider any element of the form $\neg \mathrm{P} \neg A_{j}, A_{j} \in \mathbf{A}$. This is logically equivalent to $\mathrm{O} A_{j}$, and so is implied by the conjunction $\pi \mathbf{A}$ for which $\pi^{+} \mathbf{A}=\left\{A_{j}\right\}$, and inconsistent with all the others.

Corollary 5.2. When the logic of $\mathrm{O}$ is of type EMCP, and $\mathbf{A}$ is a complete partition:

$$
\llbracket \pm \mathrm{O} \pm \mathbf{A} \rrbracket \equiv \llbracket \pm \mathrm{P} \mathbf{A} \rrbracket
$$

The corollary generalises the remarks in Section 3 on the equivalence, when $\mathrm{O}$ is of type EMCP, between Lindahl's form for normative one-agent and two-agent act positions $(8,14)$ and the forms $(11,15)$ employed in [Jones and Sergot 1992; 1993] for the same purpose.

Notice that in order to specify any element $\pi \mathbf{A}$ of $\llbracket \pm O \pm \mathbf{A} \rrbracket$ it is sufficient to specify the permissible elements $\pi^{+} \mathbf{A}$. For O of type EMCP and $\mathbf{A}$ a complete partition, $\llbracket \pm \mathrm{O} \pm \mathbf{A} \rrbracket$ can thus be represented by the set of non-empty subsets of A. (See also [Talja 1980] which takes a special case of this observation as the starting point for an algebraic treatment of the Lindahl [1977] account of 'change' of normative positions.) Notice also that when $\pi^{+} \mathbf{A}$ is a singleton, and $\mathrm{O}$ is of type EMCP (or stronger), the conjunction $\pi \mathbf{A}$ can be written equivalently in a simpler form: when $\pi^{+} \mathbf{A}=\left\{A_{j}\right\}, \pi \mathbf{A}$ is logically equivalent to $\mathrm{O} A_{j}$.

The conjunctions $\llbracket \pm \mathrm{P} \mathbf{A} \rrbracket$ have special significance for the computation of maxiconjunctions $\llbracket \pm \mathrm{O} \pm \mathbf{A} \rrbracket$ even when the logic of $\mathrm{O}$ is weaker than type EMCP. When applying the theory to the analysis of practical examples, for instance, the 
task is often to identify, for some appropriate partition $\mathbf{A}$, which of the normative positions in $\llbracket \pm \mathrm{O} \pm \mathbf{A} \rrbracket$ holds in the case at hand. Instead of generating the whole set, which may be very large, and then picking out one of the elements, it is much more efficient to divide the problem into two simpler parts: first pick out one of the conjunctions $\pi \mathbf{A}$ of $\llbracket \pm \mathbf{P} \mathbf{A} \rrbracket$; then consider just those positions consistent with $\pi \mathbf{A}$, i.e. $\llbracket \pm \mathrm{O} \pm \mathbf{A} \rrbracket / \pi \mathbf{A}$. Obviously the last step is unnecessary when $\mathrm{O}$ is of type EMCP. See [Sergot 1996] for further details.

\subsection{Refinement structures}

For $\mathrm{O}$ of type EMCP, $\mathbf{A} \geq \mathbf{B}$ implies $\llbracket \pm \mathrm{O} \pm \mathbf{A} \rrbracket \geq \llbracket \pm \mathrm{O} \pm \mathbf{B} \rrbracket$. There is much more that can be said about the structure of such maxi-conjunctions, however. We now investigate the structure of conjunctions

$$
\llbracket \pm \mathrm{O} \pm \mathbf{A} \rrbracket / \pi \mathbf{B} \quad(\mathbf{A} \geq \mathbf{B})
$$

The question is of considerable practical significance. It is the basis of the automated method to be described in Section 6 .

Suppose $B_{j} \in \pi^{+} \mathbf{B}$, i.e. $\pi \mathbf{B} \vdash \mathbf{P} B_{j}$. Since $\mathbf{A} \geq \mathbf{B}$ there is some set of elements $\mathbf{A} / B_{j}=\left\{A_{1}^{j}, \ldots, A_{m_{j}}^{j}\right\}$ such that $\vdash B_{j} \leftrightarrow\left(A_{1}^{j} \vee \ldots \vee A_{m_{j}}^{j}\right)$. By O.RE, $\vdash \mathrm{P} B_{j} \leftrightarrow$ $\mathrm{P}\left(A_{1}^{j} \vee \ldots \vee A_{m_{j}}^{j}\right)$, and when $\mathrm{O}$ is of type EMCP, $\vdash \mathrm{P} B_{j} \leftrightarrow\left(\mathrm{P} A_{1}^{j} \vee \ldots \vee \mathrm{P} A_{m_{j}}^{j}\right)$. It follows that every element $\pi \mathbf{A}$ of $\llbracket \pm \mathrm{O} \pm \mathbf{A} \rrbracket / \pi \mathbf{B}$ must have at least one $\mathrm{P} A_{i}^{j}$, i.e. every $\pi^{+} \mathbf{A}$ contains an element of $\mathbf{A} / B_{j}$.

Conversely, suppose $B_{j} \in \pi^{-} \mathbf{B}$. Then, since $\vdash A_{i}^{j} \rightarrow B_{j}$ for every $A_{i}^{j}$ in $\mathbf{A} / B_{j}$, it follows that $\vdash \neg \mathrm{P} B_{j} \rightarrow \neg \mathrm{P} A_{i}^{j}$ by the contrapositive form of rule P.RM.

TheOrem 5.3. Let $\mathbf{A}$ and $\mathbf{B}$ be complete partitions such that $\mathbf{A} \geq \mathbf{B}$. Suppose $\pi \mathbf{B}$ is an element of $\llbracket \pm \mathrm{O} \pm \mathbf{B} \rrbracket$. When $\mathrm{O}$ is of type EMCP, every element $\pi \mathbf{A}$ of $\llbracket \pm \mathrm{O} \pm \mathbf{A} \rrbracket / \pi \mathbf{B}$ must satisfy the following requirements:

(P1) for every $B_{j} \in \pi^{+} \mathbf{B}, \pi^{+} \mathbf{A}$ must include a non-empty subset of $\mathbf{A} / B_{j}$;

(P2) for every $B_{j} \in \pi^{-} \mathbf{B}$, all elements of $\mathbf{A} / B_{j}$ are in $\pi^{-} \mathbf{A}$.

Proof. Contained in the previous discussion.

Conditions (P1) and (P2) together mean that every $\pi \mathbf{A}$ of $\llbracket \pm \mathrm{O} \pm \mathbf{A} \rrbracket / \pi \mathbf{B}$ is such that:

$$
\pi^{+} \mathbf{A}=\sigma_{1}\left(\mathbf{A} / B_{1}\right) \dot{\cup} \ldots \dot{\cup} \sigma_{m}\left(\mathbf{A} / B_{m}\right)
$$

where $\sigma_{j}\left(\mathbf{A} / B_{j}\right)$ denotes a non-empty subset of $\mathbf{A} / B_{j}$ and $\dot{\cup}$ denotes disjoint union. By virtue of condition (P2), all such conjunctions can also be written equivalently in a more concise form, as follows.

Proposition 5.4. Let $\mathbf{A}$ and $\mathbf{B}$ be complete partitions such that $\mathbf{A} \geq \mathbf{B}$. Suppose $\pi \mathbf{B}$ is of the form:

$$
\neg \mathrm{P} B_{1} \wedge \ldots \wedge \neg \mathrm{P} B_{k} \wedge \mathrm{P} B_{k+1} \wedge \ldots \wedge \mathrm{P} B_{n}
$$

i.e. $\pi^{-} \mathbf{B}=\left\{B_{1}, \ldots, B_{k}\right\}$ and $\pi^{+} \mathbf{B}=\left\{B_{k+1}, \ldots, B_{n}\right\}$. When $\mathrm{O}$ is of type EMCP, every element of $\llbracket \pm \mathrm{O} \pm \mathbf{A} \rrbracket / \pi \mathbf{B}$ is logically equivalent to a conjunction of the form:

$$
\neg \mathrm{P} B_{1} \wedge \ldots \wedge \neg \mathrm{P} B_{k} \wedge \pi\left(\mathbf{A} / B_{k+1}\right) \wedge \ldots \wedge \pi\left(\mathbf{A} / B_{n}\right) .
$$

ACM Transactions on Computational Logic, Vol. 2, No. 4, October 2001. 
Example 5.5. Suppose we are given the truth of O F ( $F$ represents, let us suppose, that there is a fence between two adjoining properties) and we wish to investigate what this implies about obligations of some agent $a$. We wish to determine the normative positions of form (17) that are consistent with $\mathrm{O} F$, i.e.

$$
\llbracket \pm \mathrm{O} \pm \llbracket \pm \mathrm{E}_{a} \pm F \rrbracket \cdot \llbracket \pm F \rrbracket \rrbracket / \mathrm{O} F
$$

Proceed as follows. O $F$ can be written equivalently as $\mathrm{P} F \wedge \neg \mathrm{P} \neg F$. All conjunctions (22) will thus be equivalent to conjunctions $\neg \mathrm{P} \neg F \wedge \pi\left(\llbracket \pm \mathrm{E}_{a} \pm F \rrbracket \cdot \llbracket \pm F \rrbracket / F\right)$. Consider now $\llbracket \pm \mathrm{E}_{a} \pm F \rrbracket \cdot \llbracket \pm F \rrbracket / F=\left\{F \wedge \mathrm{E}_{a} F, F \wedge \neg \mathrm{E}_{a} F\right\} \equiv\left\{\mathrm{E}_{a} F, F \wedge \neg \mathrm{E}_{a} F\right\}$. There are three non-empty subsets of this set, and so, by Proposition 5.4, three normative positions in set (22). They are (equivalent to):

$$
\left(\begin{array}{c}
\neg \mathrm{P} \neg F \wedge \mathrm{P} \mathrm{E}_{a} F \wedge \neg \mathrm{P}\left(F \wedge \neg \mathrm{E}_{a} F\right) \\
\neg \mathrm{P} \neg F \wedge \neg \mathrm{P} \mathrm{E}_{a} F \wedge \mathrm{P}\left(F \wedge \neg \mathrm{E}_{a} F\right) \\
\neg \mathrm{P} \neg F \wedge \mathrm{P} \mathrm{E}_{a} F \wedge \mathrm{P}\left(F \wedge \neg \mathrm{E}_{a} F\right)
\end{array}\right) \equiv\left(\begin{array}{c}
\mathrm{O} \mathrm{E}_{a} F \\
\mathrm{O} F \wedge \neg \mathrm{P}_{a} F \\
\mathrm{O} F \wedge \mathrm{P} \mathrm{E}_{a} F \wedge \mathrm{P} \neg \mathrm{E}_{a} F
\end{array}\right)
$$

In similar fashion we may calculate which of the 'collectivistic' normative positions of form (19) for two agents $a$ and $b$ are consistent with, say $\mathrm{O}_{a} F$ :

$$
\begin{aligned}
\llbracket \pm \mathrm{O} \pm \llbracket \pm\left(\begin{array}{c}
\mathrm{E}_{a} \\
\mathrm{E}_{b}
\end{array}\right) \pm F \rrbracket \cdot \llbracket \pm F \rrbracket \rrbracket / \mathrm{O}_{a} F & = \\
& \llbracket \pm \mathrm{O} \pm \llbracket \pm \mathrm{E}_{a} \pm F \rrbracket \cdot \llbracket \pm \mathrm{E}_{b} \pm F \rrbracket \cdot \llbracket \pm F \rrbracket \rrbracket / \mathrm{O}_{a} F
\end{aligned}
$$

These positions will be (equivalent to) conjunctions of the form $\mathrm{OE}_{a} F \wedge C$ : to determine $C$ we need to consider

$$
\begin{aligned}
\llbracket \pm \mathrm{E}_{a} \pm F \rrbracket \cdot \llbracket \pm \mathrm{E}_{b} \pm F \rrbracket \cdot \llbracket \pm F \rrbracket / \mathrm{E}_{a} F & =\left(\llbracket \pm \mathrm{E}_{a} \pm F \rrbracket / \mathrm{E}_{a} F\right) \cdot\left(\llbracket \pm \mathrm{E}_{b} \pm F \rrbracket / \mathrm{E}_{a} F\right) \\
& =\left\{\mathrm{E}_{a} F \wedge \mathrm{E}_{b} F, \mathrm{E}_{a} F \wedge \neg \mathrm{E}_{b} F\right\} .
\end{aligned}
$$

There are three non-empty subsets, and so again three normative positions of the form we seek. They are (equivalent to):

$$
\left(\begin{array}{c}
\mathrm{O} \mathrm{E}_{a} F \wedge \mathrm{PE}_{b} F \wedge \neg \mathrm{P} \neg \mathrm{E}_{b} F \\
\mathrm{OE}_{a} F \wedge \neg \mathrm{PE}_{b} F \wedge \mathrm{P} \neg \mathrm{E}_{b} F \\
\mathrm{O} \mathrm{E}_{a} F \wedge \mathrm{P} \mathrm{E}_{b} F \wedge \mathrm{P} \neg \mathrm{E}_{b} F
\end{array}\right) \equiv\left(\begin{array}{c}
\mathrm{OE}_{a} F \wedge \mathrm{O} \mathrm{E}_{b} F \\
\mathrm{O} \mathrm{E}_{a} F \wedge \mathrm{O} \neg \mathrm{E}_{b} F \\
\mathrm{OE}_{a} F \wedge \mathrm{P} \mathrm{E}_{b} F \wedge \mathrm{P} \neg \mathrm{E}_{b} F
\end{array}\right)
$$

The procedure is quite mechanical, and readily automated, as described in Section 6 .

The example also illustrates an important advantage of basing the generation of normative positions on cumulative fact/act positions of the form $\llbracket \pm \mathrm{E}_{a} \pm F \rrbracket \cdot \llbracket \pm F \rrbracket$ in preference to the simpler act positions $\llbracket \pm \mathrm{E}_{a} \pm F \rrbracket$ employed by Lindahl. Not only is the resulting analysis more precise, but Lindahl's act positions are not a refinement of $\llbracket \pm F \rrbracket$ and so the computational methods just described cannot be exploited, except in a messy and rather indirect way.

This leads naturally to the second of the questions raised at the beginning of this section, and specifically, to the question of what act positions $\mathbf{A}$ yield the most refined, or most appropriate, set of normative positions $\llbracket \pm \mathrm{O} \pm \mathbf{A} \rrbracket$.

\subsection{Maximally refined positions: Normal forms}

For $\mathrm{O}$ of type $E M C P, \llbracket \pm \mathrm{O} \pm \mathbf{A} \rrbracket$, and hence $\llbracket \pm \mathrm{P} \mathbf{A} \rrbracket$, is the most refined set of normative positions that can be constructed on the basis of a partition $\mathbf{A}$, in the 
following sense. Suppose $\Phi$ is a set of sentences closed under subsentences (if $B$ is in $\Phi$ then all subsentences of $B$ are also in $\Phi$ ). Suppose further that $\mathbf{A}$ is a partition such that all sentences of $\Phi$, and hence all boolean compounds of sentences of $\Phi$, are expressible as disjunctions of sentences of $\mathbf{A}$. Then, for $\mathrm{O}$ of type $E M C P$, every boolean compound of sentences $O B, B$ in $\Phi$, and all their subsentences, is logically equivalent to a disjunction of sentences from the following partition:

$$
\llbracket \pm \mathrm{O} \pm \mathbf{A} \rrbracket \cdot \mathbf{A} \cdot \llbracket \pm \mathrm{O} \top \rrbracket
$$

If the logic of $\mathrm{O}$ is strengthened to be of the same type as Standard Deontic Logic, a normal modal logic of type $K D=E M C N P$, then the last component $\llbracket \pm \mathrm{O} \top \rrbracket$ can be omitted, since then $\llbracket \pm O \top \rrbracket=\{\mathrm{O} \top\}=\{\top\}$. Expression (23) provides a disjunctive normal form for the fragment of the logic consisting of sentences $\mathrm{O} B$, $B$ in $\Phi$, and their subsentences.

Let $\mathbf{P} \times \mathbf{Q}$ stand for the set of pairwise, not necessarily consistent, conjunctions that may be formed by conjoining a sentence from $\mathbf{P}$ with a sentence from $\mathbf{Q}$. Then clearly, by definition:

$$
\llbracket \pm \mathrm{O} \pm \mathbf{A} \rrbracket \cdot \mathbf{A} \cdot \llbracket \pm \mathrm{O} \top \rrbracket \subseteq \llbracket \pm \mathrm{O} \pm \mathbf{A} \rrbracket \times \mathbf{A} \times \llbracket \pm \mathrm{O} \top \rrbracket .
$$

However, since for $\mathrm{O}$ of type $E M C P$ there are no logical relationships between expressions in the set $\llbracket \pm \mathrm{O} \pm \mathbf{A} \rrbracket$ and the expressions $\mathbf{A}$, then:

$$
\llbracket \pm \mathrm{O} \pm \mathbf{A} \rrbracket \cdot \mathbf{A} \cdot \llbracket \pm \mathrm{O} \top \rrbracket=\llbracket \pm \mathrm{O} \pm \mathbf{A} \rrbracket \times \mathbf{A} \times \llbracket \pm \mathrm{O} \top \rrbracket .
$$

Thus, there is nothing interesting to be said about the structure of positions (23); the essential structure is that of the component $\llbracket \pm O \pm \mathbf{A} \rrbracket$. In this sense, $\llbracket \pm O \pm \mathbf{A} \rrbracket$ is the most refined set of normative positions that can be constructed on the basis of the partition A. Of course it is possible to employ (23) in the construction of other classes of meaningful normative positions, such as:

$$
\llbracket \pm \mathrm{O} \pm \llbracket \pm \mathrm{O} \pm \mathbf{A} \rrbracket \cdot \mathbf{A} \cdot \llbracket \pm \mathrm{O} \top \rrbracket \rrbracket .
$$

We shall not investigate such positions in this paper.

What of the act positions? Which act positions A yield the most refined set of normative positions $\llbracket \pm \mathrm{O} \pm \mathbf{A} \rrbracket$ ? Here the answer is more complicated (or rather longer) because it depends on the properties of the action modalities employed. Full discussion of the possibilities is omitted for lack of space.

In the remainder of the paper, and in the automated system to be described in the next section, we restrict attention to act positions of a particular kind: we consider only act expressions containing elementary propositional variables or their negations within the scope of an action operator. We thereby rule out of consideration act expressions such as $\mathrm{E}_{a}(p \wedge q), \mathrm{E}_{a}(p \wedge \neg q), \mathrm{E}_{a}(p \vee q)$, and so on. We impose this restriction purely for practical reasons. In principle there is nothing problematic about allowing the more general forms of act expressions, and then constructing the most refined set of act positions and the most refined set of normative act positions; in practice, it is not clear that the added level of precision is worth the extra trouble.

So, as a practical matter, in the automated system to be described next, the most refined act positions to be considered for agents $a, b, \ldots$ and propositional variables 
$p, q, \ldots$, are those of the form:

$$
\left\langle \pm\left(\begin{array}{c}
\mathrm{E}_{a} \\
\mathrm{E}_{b} \\
\vdots
\end{array}\right) \pm\left(\begin{array}{c}
p \\
q \\
\vdots
\end{array}\right)\right\rangle \stackrel{\text { def }}{=} \llbracket \pm\left(\begin{array}{c}
\mathrm{E}_{a} \\
\mathrm{E}_{b} \\
\vdots
\end{array}\right) \pm\left(\begin{array}{c}
p \\
q \\
\vdots
\end{array}\right) \rrbracket \cdot \llbracket \pm\left(\begin{array}{c}
p \\
q \\
\vdots
\end{array}\right) \rrbracket
$$

It is convenient to have a more concise notation for these act positions. We write also:

$$
\Psi[a, b, \ldots ; F, G, \ldots] \stackrel{\text { def }}{=}\left\langle \pm\left(\begin{array}{c}
\mathrm{E}_{a} \\
\mathrm{E}_{b} \\
\vdots
\end{array}\right) \pm\left(\begin{array}{c}
F \\
G \\
\vdots
\end{array}\right)\right\rangle
$$

$F, G, \ldots$ will usually be elementary propositional variables. It may be helpful to note that $\Psi[a, b, \ldots ; F, G, \ldots]$ can be written equivalently (Theorem 3.1 ) in the following ways:

$$
\begin{aligned}
\Psi & {[a, b, \ldots ; F, G, \ldots] } \\
& =\llbracket \pm F \rrbracket \cdot \llbracket \pm \mathrm{E}_{a} \pm F \rrbracket \cdot \llbracket \pm \mathrm{E}_{b} \pm F \rrbracket \cdot \ldots \cdot \llbracket \pm G \rrbracket \cdot \llbracket \pm \mathrm{E}_{a} \pm G \rrbracket \cdot \llbracket \pm \mathrm{E}_{b} \pm G \rrbracket \cdot \ldots \\
& =\Psi[a, b, \ldots ; F] \cdot \Psi[a, b, \ldots ; G] \cdot \ldots \\
& =\Psi[a ; F, G, \ldots] \cdot \Psi[b ; F, G, \ldots] \cdot \ldots \\
& =\Psi[a ; F] \cdot \Psi[b ; F] \cdot \ldots \cdot \Psi[a ; G] \cdot \Psi[b ; G] \cdot \ldots
\end{aligned}
$$

When $\mathcal{A} g$ denotes a set of agent names and $\mathcal{P}$ rops a set of propositional variables we shall also write $\Psi[\mathcal{A} g ; \mathcal{P}$ rops $]$.

\section{AUTOMATION: NORMAN-G}

The procedures described in previous sections have been implemented in a computer program, Norman-G, a prototype system intended to facilitate application of the theory to the analysis of practical examples, either for the purpose of interpretation and disambiguation of legal texts, rules, and regulations, or in the design and specification of a new set of norms. A typical example is the case discussed in [Jones and Sergot 1992; 1993] concerning access 'rights' to sensitive medical information in a hospital database [Ting 1990]. The problem here is to clarify and expand an incomplete and very imprecise statement of requirements into a precise specification at some desired level of detail.

In order to conduct such an analysis, the general strategy is to pick some scheme $\llbracket \pm \mathrm{O} \pm \mathbf{A} \rrbracket$ which represents the problem under consideration at the appropriate level of detail. We refer to this as the target partition. In Norman-G the partition A is always a set of act positions of the form $\Psi[\mathcal{A} g ; \mathcal{P}$ rops $], \mathcal{A} g$ a set of agent names and $\mathcal{P}$ rops a set of propositional variables. The objective of the analysis is to identify which position in the target partition holds in the (real or hypothetical) circumstances under consideration. In practice, there will often be points of detail on which the user will be unable or unwilling to decide. In that case the result of the analysis will be a disjunction of positions.

The number of positions in the target partition $\llbracket \pm O \pm \mathbf{A} \rrbracket$ when $\mathrm{O}$ is of type $E M C P$ is $2^{|\mathbf{A}|}-1$. When $\mathbf{A}$ is of the form $\Psi[\mathcal{A} g ; \mathcal{P}$ rops $]$ and there are $m$ agents in $\mathcal{A} g$ and $n$ propositional variables in $\mathcal{P}$ rops, the number of act positions in $\Psi[\mathcal{A} g ; \mathcal{P}$ rops $]$ 


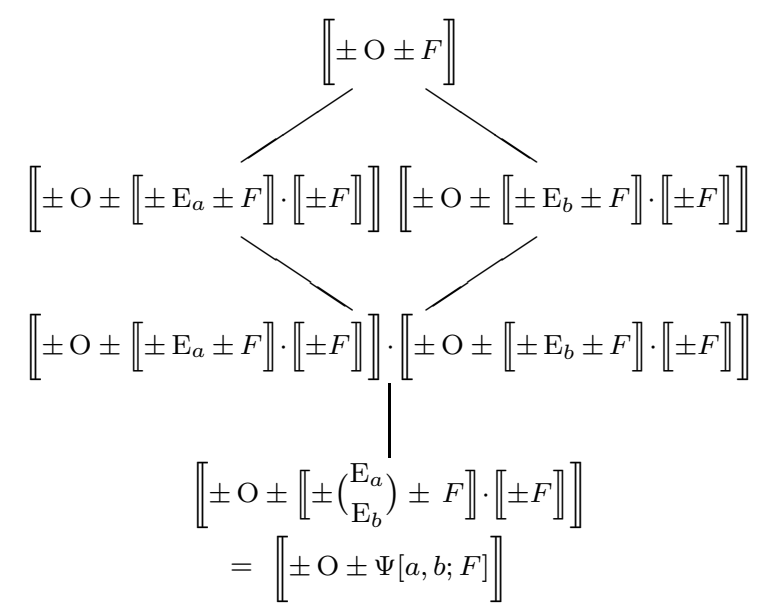

Fig. 4. Positions for two agents $a$ and $b$ and one state of affairs $F$

is $2^{(m+1) n}$. The number of normative positions in the target partition is then $2^{2^{(m+1) n}}-1$. Although it is easy to write a computer program to generate all these expressions, it would obviously be a nonsense to present the user with the complete list and then ask which one applies. Estimate how long it would take to examine even a small example: scanning the list of positions for 2 agents and 2 propositions at, say, 10 positions per second would still take 58 billion $\left(58 \times 10^{9}\right)$ years to go through the entire list.

It is practical however to perform such an analysis if it is conducted by a process of progressive refinement. At each stage the analysis completed so far is used to constrain the choice of possible positions at the next level of detail. Given a target partition $\llbracket \pm \mathrm{O} \pm \mathbf{A} \rrbracket$, find a sequence of refinements $\mathbf{A}_{0} \leq \mathbf{A}_{1} \leq \ldots \leq \mathbf{A}_{N} \leq \mathbf{A}$ and proceed as follows. First determine which position $\pi_{0} \mathbf{A}_{0}$ of $\llbracket \pm \mathrm{O} \pm \mathbf{A}_{0} \rrbracket$ holds in the given circumstance. Then consider the candidate positions at the next level of detail: determine position $\pi_{1} \mathbf{A}_{1}$ from the candidate set $\llbracket \pm O \pm \mathbf{A}_{1} \rrbracket / \pi_{0} \mathbf{A}_{0}$. Now consider $\llbracket \pm \mathrm{O} \pm \mathbf{A}_{2} \rrbracket / \pi_{1} \mathbf{A}_{1}$, and so on, until left with the task of identifying a position from the target partition, which will be an element of $\llbracket \pm \mathrm{O} \pm \mathbf{A} \rrbracket / \pi_{N} \mathbf{A}_{N}$. As described in the previous section, the calculation of the candidate positions at each individual step is simple (especially when $\mathrm{O}$ is of type EMCP) and quite mechanical. Section 7 presents a small worked example.

In practice the procedure is more complicated because usually it will not be a sequence of refinements that has to be considered but a more elaborate structure. Figure 4 shows the refinement structure in Norman-G for the case of two agents $a$ and $b$ and one state of affairs $F$. Figure 5 shows the structure for the case $\mathcal{A} g=\{a\}$ and $\mathcal{P}$ rops $=\{F, G\}$. In each case, the analysis would begin with the partitions at the top of the figure and work its way down to the more refined partitions shown lower down.

The general case is difficult to draw, but is readily described: since partition ACM Transactions on Computational Logic, Vol. 2, No. 4, October 2001. 


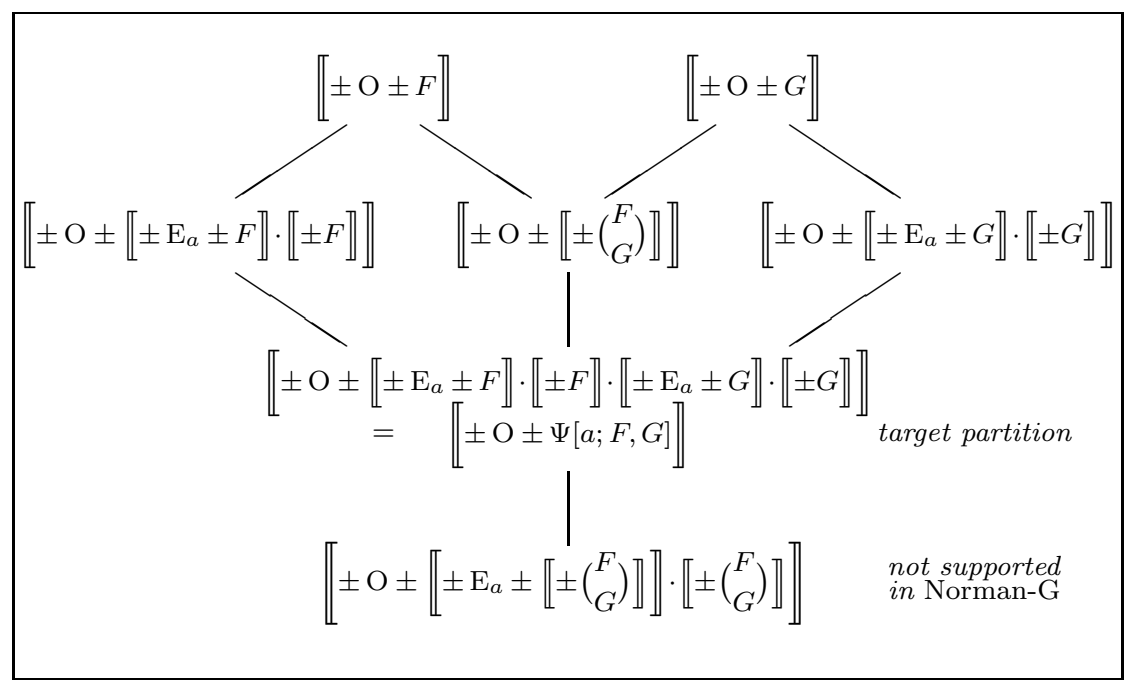

Fig. 5. Positions for one agent $a$ and two states of affairs $F$ and $G$

$\llbracket \pm \mathrm{O} \pm \Psi[\mathcal{A} ; \mathcal{P}] \rrbracket \geq \llbracket \pm \mathrm{O} \pm \Psi\left[\mathcal{A}^{\prime} ; \mathcal{P}^{\prime}\right] \rrbracket$ when $\mathcal{A}^{\prime} \subseteq \mathcal{A}$ and $\mathcal{P}^{\prime} \subseteq \mathcal{P}$, the structure is (almost exactly) that of the lattice of subsets of $\mathcal{A} g \cup \mathcal{P}$ rops. There are various ways of depicting such a lattice.

Automation of the position-generating and inference procedures of Section 5 is now straightforward: the procedures can be reduced to a very simple graphcolouring algorithm, readily implemented in a computer program. We just describe the basic case for $\mathrm{O}$ of type EMCP. Weaker logics require an additional mechanism (see the remarks at the end of Section 5.1) not implemented in the current version of Norman-G.

Suppose $\Psi[\mathcal{A} ; \mathcal{P}]$ is one of the act positions in the refinement structure for $\llbracket \pm \mathrm{O} \pm \Psi[\mathcal{A} g ; \mathcal{P}$ rops $] \rrbracket$, as described above. Every sentence of every $\Psi[\mathcal{A} ; \mathcal{P}]$ is represented as a node in a graph. Edges of the graph correspond to implications between these sentences. These implications can be determined from the structure of the sentences and so are generated automatically. Figure 6 shows a small fragment of the graph, where $p$ is in $\mathcal{P}$ rops and $a$ and $b$ are in $\mathcal{A} g$.

Now: every normative position can be represented as a colouring of this graph. For suppose that $\mathbf{A}^{\prime}=\left\{A_{1}, \ldots, A_{n}\right\}$ is one of the sets of act positions $\Psi[\mathcal{A} ; \mathcal{P}]$. Then every conjunction $\pi \mathbf{A}^{\prime}$ of $\llbracket \pm \mathrm{O} \pm \mathbf{A}^{\prime} \rrbracket$ can be represented as a colouring of the nodes $A_{i}$ of $\mathbf{A}^{\prime}$ : blue (say) when $\mathrm{P} A_{i}$ is in $\pi \mathbf{A}^{\prime}$ and red when $\neg \mathrm{P} A_{i}$ is in $\pi \mathbf{A}^{\prime}$. A plain (uncoloured) node $A_{i}$ represents the (tautologous) disjunction $\mathrm{P} A_{i} \vee \neg \mathrm{P} A_{i}$. For the graph as a whole, the colouring must respect the constraints imposed by conditions (P1) and (P2) of Theorem 5.3: when node $A_{i}$ is blue ('permissible'), all nodes implied by $A_{i}$ must be blue; when node $A_{i}$ is red ('prohibited'), all nodes implying $A_{i}$ - those in the refinements of $A_{i}$-must also be red; no partition $\Psi[\mathcal{A} ; \mathcal{P}]$ and no partition of a blue node may have all nodes red. A partially coloured graph represents a disjunction of positions. A position of the target partition $\llbracket \pm \mathrm{O} \pm \Psi[\mathcal{A} g ;$ Props $] \rrbracket$ is uniquely identified when all nodes in $\Psi[\mathcal{A} g ;$ Props $]$ are coloured red or blue; by virtue of conditions (P1) and (P2), the graph of the 


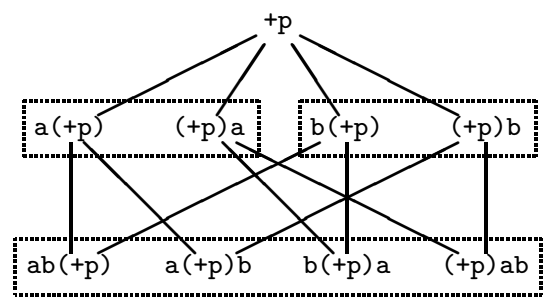

Node labels use a shorthand to save space: 'active' agents are listed on the left of the label, 'passive' agents on the right. So e.g. node label $\mathrm{a}(+\mathrm{p}) \mathrm{b}$ is shorthand for $\mathrm{E}_{a} p \wedge \neg \mathrm{E}_{b} p$; (+p) ab is shorthand for $p \wedge \neg \mathrm{E}_{a} p \wedge \neg \mathrm{E}_{b} p$, etc. The edges of the graph correspond to implications (all directed upwards on the figure). The dotted lines signify partitions (of $p$ in this example).

Fig. 6. A fragment of a Norman-G graph

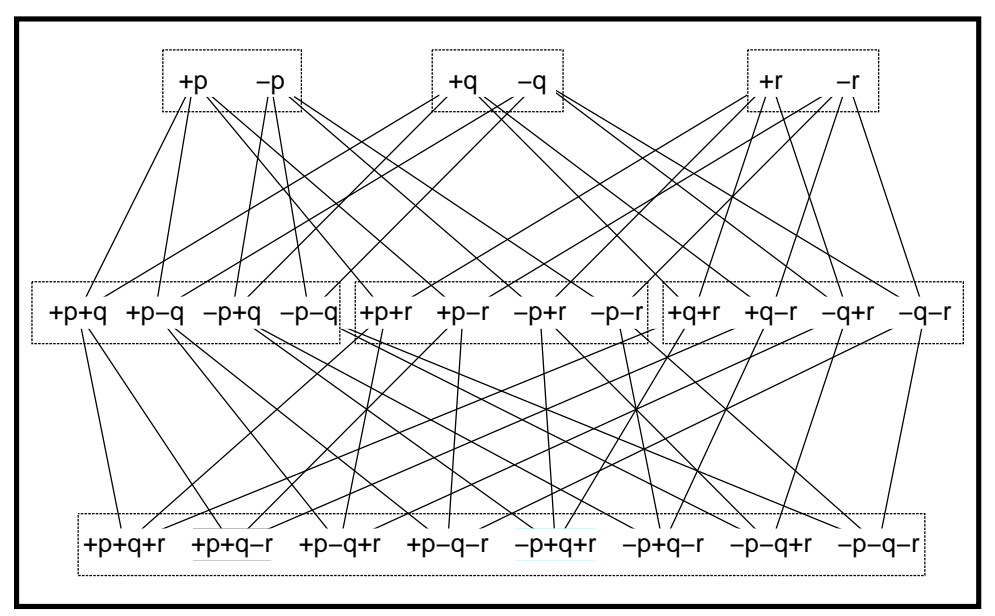

Node labels use a shorthand to save space: e.g. the node label $+p-q+r$ is shorthand for the conjunction $p \wedge \neg q \wedge r$. There are no agents depicted in the top-level display.

Fig. 7. Top-level Norman-G display (three propositional variables)

whole refinement structure will then be completely coloured too.

The user interface for Norman-G is constructed around a display of this graph. There are facilities for viewing selected portions of the graph and for manipulating the nodes (colouring them). The basic operation is to colour a selected node blue or red. The propagation of colours through the graph provides a visualization of the inference mechanisms (in a way that pre-supposes no familiarity with the underlying theory). Figure 7 shows a reconstruction of the top-level Norman-G display for a scenario containing three propositional variables.

There are similar displays of the act positions for the various agents and combinations of agents. Figure 6 shows part of such a display. Several fragments can be displayed at once; the user switches between them as desired.

Figure 8 shows how the graph of Figure 7 looks when some of the nodes have ACM Transactions on Computational Logic, Vol. 2, No. 4, October 2001. 


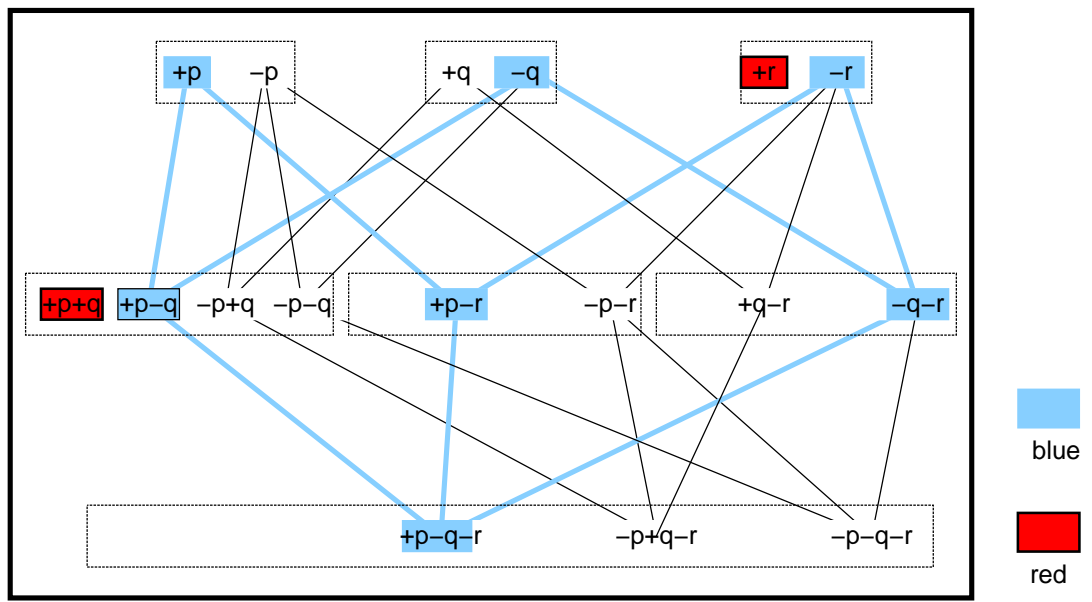

Fig. 8. Display of a partially coloured Norman-G graph

been coloured. (Different shades are used to distinguish nodes that were coloured by the user from those whose colour was inferred but this is difficult to reproduce clearly here.) When a node is coloured red by the user, all newly inferred red nodes are first displayed to show the propagation of colours, and then the inferred red nodes are deleted from the diagram to reduce clutter; of the red nodes, only the explicitly coloured ones remain. All blue nodes, explicitly coloured and inferred, remain on the display.

The deletion of inferred red nodes from the graph is important. It can be seen as a visual rendition of Proposition 5.4. It is of practical significance, because it allows large portions of the graph to be pruned away entirely: the more explicitly coloured red nodes, the smaller the graph that has to be maintained.

Norman-G can also be used in dialogue mode: the system generates a series of questions to the user by going through the (uncoloured) nodes in a pre-determined order, simplest to most complex. The graph display then functions as a trace of the dialogue.

There are three further features of Norman-G that are important for practical purposes but which we do not describe in detail here for lack of space.

(1) The language is extended with another (alethic) modality, $\square$ and $\diamond$ for 'necessary' and 'possible', respectively, which acts as an additional filter on the set of fact/act positions to be considered. $\square$ is of type $S 5$ (KT5 in the standard classification), with the further property $\square A \rightarrow \mathrm{O} A$. This additional modality is useful for three main purposes:

(a) It can be used to rule out certain fact combinations and act expressions as either uninteresting or impossible, in much the same way that integrity constraints are used in databases and knowledge representation. This is important because the number of positions to be considered is thereby significantly reduced.

(b) It can be used to focus attention on fact/act combinations of particular interest. For example, including (a representation of) the schema $\square \mathrm{E}_{a} A$ excludes from 
consideration all cases where agent $a$ does not act.

(c) It can be used as a way of strengthening the underlying logic. For instance, it may be desirable, for certain purposes, to adopt an additional schema $\mathrm{E}_{x} A \rightarrow$ $\neg \mathrm{E}_{y} A$, for all pairs of agents $x \neq y$. The effect is obtained by including (a representation of $) \neg \diamond\left(\mathrm{E}_{x} A \wedge \mathrm{E}_{y} A\right)(x \neq y)$ in the scenario specification.

Implementation of this additional modality is very straightforward: $\neg \diamond$ nodes (coloured black in Norman-G) delete portions of the graph in (almost exactly) the same way as $\neg \mathrm{P}$ (red) nodes do. Details omitted here.

(2) It is convenient to have in the language a separate sort of exogenous propositional variables. These represent states of affairs that cannot meaningfully be brought about by an agent (it is raining, it is the weekend, ...). The meaningless act expressions could also be eliminated by means of the $\square$ modality just discussed, but the requirement is so common that a special sort of propositional variable is more natural.

(3) It is possible to specify certain pairs of agent-act combinations as independent of one another. This allows the Norman-G graph to be decomposed into independent parts, and reduces drastically the number of positions to be considered. It is critical for practical application to realistic examples. We do not have the space here for a full discussion. Roughly: the (states of affairs represented by) sentences $A$ and $B$ are normatively independent when $\mathrm{P}(A \wedge X) \wedge \mathrm{P}(B \wedge X) \rightarrow \mathrm{P}(A \wedge B \wedge X)$ for all sentences $X$. Notice that every node in the sub-graph rooted at $A \wedge B$ is logically equivalent to $A \wedge B \wedge X$ for some $X$. But, in Norman-G, $A \wedge X$ and $B \wedge X$ are then also (logically equivalent to) nodes in the sub-graphs rooted at $A$ and $B$ respectively. So, when $A$ and $B$ are normatively independent, the two sub-graphs rooted at $A$ and $B$ can be coloured (analysed) independently without having to consider possible interactions between the colourings of their nodes: the colouring of the node $A \wedge B \wedge X$ is already determined because $\mathrm{P}(A \wedge B \wedge X) \leftrightarrow \mathrm{P}(A \wedge X) \wedge \mathrm{P}(B \wedge X)$. Norman-G provides facilities for declaring which combinations of formulas are normatively independent in this sense, and for dividing a partially coloured graph into separate parts when this becomes evident during the course of an analysis.

\section{EXAMPLE}

We present here a small example to demonstrate how the theory and the Norman-G system can be used in practice. The example is for illustration only; there are many features of the example we shall not be able to discuss here. A slightly longer and more detailed account is given in [Sergot and Richards 2000].

The example is a modified version of the car park example in [Lee 1988] that was discussed briefly in Section 2. It concerns the specification of which categories of staff are permitted and not permitted to park in a car park. We choose it because it is familiar and requires no further explanation. In Lee's example, administrators are permitted to park in the car park. We ignore other categories of staff here.

Consider the following scenario:

$a$ is an administrator, permitted to park in the car park. $a$ has two cars, $c a r-a_{1}$ and $c a r-a_{2} . b$ is a disgraced administrator, banned from the car park. $b$ has one car, car-b. $c$ is a passer-by. $g$ is the gatekeeper, charged with controlling access to the car park and ensuring the rules are obeyed. 
We shall not attempt in this paper to cover every feature of the example. In particular the representation of what it means to say that the gatekeeper $g$ is responsible for ensuring that the rules of the car park are obeyed raises a number of difficult points which are outside the scope of this paper.

Let $p\left(a_{1}\right), p\left(a_{2}\right), p(b)$ represent that cars car- $a_{1}, c a r-a_{2}, c a r-b$ are parked in the car park, respectively. We take it that the following at least is implicit and obvious from the scenario description as given above: that it is not permitted that $c a r-b$ is parked in the car park, $\neg \mathrm{P} p(b)$; that it is permitted but not obligatory that car- $a_{1}$ is parked in the car park, $\mathrm{P} p\left(a_{1}\right) \wedge \mathrm{P} \neg p\left(a_{1}\right)$; and that it is permitted but not obligatory that car- $a_{2}$ is parked in the car park, $\mathrm{P} p\left(a_{2}\right) \wedge \mathrm{P} \neg p\left(a_{2}\right)$.

What else holds according to the rules of the car park (as we imagine them to be from the scenario and previous experience of typical car parks)? In order to investigate the possibilities in a systematic fashion, and to identify any points requiring further clarification, the task is to pick out one or, in the case of some residual uncertainty, several of the positions from the following scheme:

$$
\llbracket \pm \mathrm{O} \pm \Psi\left[a, b, c, g ; p\left(a_{1}\right), p\left(a_{2}\right), p(b)\right] \rrbracket=\llbracket \pm \mathrm{O} \pm\left\langle \pm\left(\begin{array}{c}
\mathrm{E}_{a} \\
\mathrm{E}_{b} \\
\mathrm{E}_{c} \\
\mathrm{E}_{g}
\end{array}\right) \pm\left(\begin{array}{c}
p\left(a_{1}\right) \\
p\left(a_{2}\right) \\
p(b)
\end{array}\right)\right\rangle \rrbracket
$$

This is the target partition for this exercise. We want to restrict attention to those positions in the target partition that are consistent with the initial assertions:

$$
\neg \mathrm{P} p(b) \wedge\left(\mathrm{P} p\left(a_{1}\right) \wedge \mathrm{P} \neg p\left(a_{1}\right)\right) \wedge\left(\mathrm{P} p\left(a_{2}\right) \wedge \mathrm{P} \neg p\left(a_{2}\right)\right)
$$

The dynamic behaviour of the graphical interface is difficult to illustrate in text. Instead we show the operation of Norman-G in dialogue mode, whereby the user, having coloured parts of the graph to represent initial assertions, responds to questions generated by the program. The user is able to postpone giving an answer, or to terminate the dialogue at any time.

We now show the transcript of such a dialogue. To keep the illustration manageable we ignore the agents $c$ and $g$ and consider the simpler target partition:

$$
\llbracket \pm \mathrm{O} \pm \Psi\left[a, b ; p\left(a_{1}\right), p\left(a_{2}\right), p(b)\right] \rrbracket=\llbracket \pm \mathrm{O} \pm\left\langle \pm\left(\begin{array}{c}
\mathrm{E}_{a} \\
\mathrm{E}_{b}
\end{array}\right) \pm\left(\begin{array}{c}
p\left(a_{1}\right) \\
p\left(a_{2}\right) \\
p(b)
\end{array}\right)\right\rangle \rrbracket
$$

The dialogue for the original target partition (24) proceeds in exactly the same way though is obviously longer than the one shown here.

The left hand column of the transcript shows the question generated by the Norman-G program and the answer given in response. The right hand column shows the corresponding assertion that is made and displayed on the graph. The three expressions above the horizontal line are the initial assertions made before the dialogue is invoked. (The dialogue can also be invoked with an empty set of initial assertions, or at any other point of colouring the graph.) 


\begin{tabular}{|c|c|c|c|}
\hline & & $\begin{array}{r}\neg \mathrm{P} p(b) \\
\mathrm{P} p\left(a_{1}\right) \wedge \mathrm{P} \neg p\left(a_{1}\right) \\
\mathrm{P} p\left(a_{2}\right) \wedge \mathrm{P} \neg p\left(a_{2}\right)\end{array}$ & \\
\hline $\mathrm{P}\left(p\left(a_{1}\right) \wedge p\left(a_{2}\right)\right) ?$ & $-\mathrm{n}$ & $\neg \mathrm{P}\left(p\left(a_{1}\right) \wedge p\left(a_{2}\right)\right)$ & Note [1] \\
\hline $\mathrm{P}\left(\neg p\left(a_{1}\right) \wedge \neg p\left(a_{2}\right)\right) ?$ & $-\mathrm{y}$ & $\mathrm{P}\left(\neg p\left(a_{1}\right) \wedge \neg p\left(a_{2}\right)\right)$ & \\
\hline $\mathrm{P} \mathrm{E}_{a} p\left(a_{1}\right) ?$ & $-\mathrm{y}$ & $\mathrm{P} \mathrm{E}_{a} p\left(a_{1}\right)$ & \\
\hline $\mathrm{P}\left(p\left(a_{1}\right) \wedge \neg \mathrm{E}_{a} p\left(a_{1}\right)\right) ?$ & $-\mathrm{n}$ & $\neg \mathrm{P}\left(p\left(a_{1}\right) \wedge \neg \mathrm{E}_{a} p\left(a_{1}\right)\right)$ & Note [2] \\
\hline $\mathrm{P} \mathrm{E}_{a} \neg p\left(a_{1}\right) ?$ & $-\mathrm{y}$ & $\mathrm{P} \mathrm{E}_{a} \neg p\left(a_{1}\right)$ & \\
\hline $\mathrm{P}\left(\neg p\left(a_{1}\right) \wedge \neg \mathrm{E}_{a} \neg p\left(a_{1}\right)\right) ?$ & $-\mathrm{n}$ & $\neg \mathrm{P}\left(\neg p\left(a_{1}\right) \wedge \neg \mathrm{E}_{a} \neg p\left(a_{1}\right)\right)$ & \\
\hline $\mathrm{P} \mathrm{E}_{b} p\left(a_{1}\right) ?$ & $-\mathrm{n}$ & $\neg \mathrm{P} \mathrm{E}_{b} p\left(a_{1}\right)$ & Note [3] \\
\hline $\mathrm{P} \mathrm{E}_{b} \neg p\left(a_{1}\right) ?$ & $-\mathrm{n}$ & $\neg \mathrm{P} \mathrm{E}_{b} \neg p\left(a_{1}\right)$ & \\
\hline Treat $p\left(a_{2}\right)$ like $p\left(a_{1}\right)$ & & $\mathrm{P} \mathrm{E}_{a} p\left(a_{2}\right)$ & Note [4] \\
\hline & & $\neg \mathrm{P}\left(p\left(a_{2}\right) \wedge \neg \mathrm{E}_{a} p\left(a_{2}\right)\right)$ & \\
\hline & & $\mathrm{P} \mathrm{E}_{a} \neg p\left(a_{2}\right)$ & \\
\hline & & $\neg \mathrm{P}\left(\neg p\left(a_{2}\right) \wedge \neg \mathrm{E}_{a} \neg p\left(a_{2}\right)\right)$ & \\
\hline & & $\neg \mathrm{P} \mathrm{E}_{b} p\left(a_{2}\right)$ & \\
\hline & & $\neg \mathrm{P} \mathrm{E}_{b} \neg p\left(a_{2}\right)$ & \\
\hline $\mathrm{P}_{a} \neg p(b) ?$ & $-\mathrm{n}$ & $\neg \mathrm{P} \mathrm{E}_{a} \neg p(b)$ & Note [5] \\
\hline
\end{tabular}

Note [1]. We are assuming that it would not be permitted for both of administrator $a$ 's cars to be parked at the same time. This would need to be checked with the car park authorities, or left undetermined if it were not regarded as important. One purpose of the analysis to identify points of detail that may have remained undetected otherwise. Note that Norman-G does not force a decision on such questions - they can be left unanswered. When dealing with legislative texts, in particular, it will often be the case that some points of detail are deliberately left ambiguous by the legislator. That is perfectly compatible with the use of Norman-G. The aim is to eliminate points of inadvertent ambiguity.

Note [2]. We are supposing for the purpose of the example that if one of the administrator $a$ 's cars is parked, then $a$ must be at least one of those responsible for the car's being parked, i.e., that $\mathrm{O}\left(p\left(a_{1}\right) \rightarrow \mathrm{E}_{a} p\left(a_{1}\right)\right)$ holds. It might be tempting to read this as saying that if $c a r-a_{1}$ is parked then it must have been the administrator $a$ who parked it. But note that expression $\mathrm{E}_{a} p\left(a_{1}\right)$ does not necessarily signify that $a$ parks $c a r-a_{1} ; a$ may bring about $p\left(a_{1}\right)$ in some different way, perhaps even unintentionally. See [Sergot and Richards 2000] for further discussion of this point.

Note [3]. One might suppose that the answer to this question is already implied by answers given earlier, in particular at Note [2]. This is not so: $\mathrm{E}_{a} F$ does not necessarily imply $\neg \mathrm{E}_{b} F$ for other agents $b \neq a$. $a$ and $b$ could act jointly to bring about $F$, or could even act unintentionally in such a way that each brings about $F$. We have answered this question in the negative since it seems right to say that the banned administrator $b$ is not permitted to park $a$ 's car and it shortens the dialogue. We comment further on this point below. See also the discussion of the 
'single agent assumption' in [Sergot and Richards 2000].

Note [4]. Norman-G provides a range of similar shortcuts for the user's convenience. (The actual syntax is slightly different from that shown here.) Another common shortcut, for example, is to specify that expressions containing agents $a$ and $b$, or specific patterns of such expressions, are to be treated in the same way: what holds for $a$ holds for $b$, and vice versa. These are not features of the language but merely shorthand devices for entering commonly occurring patterns of assertions.

Note [5]. The answer to this question is far from obvious. We will assume it is 'no' for the purpose of the example. Similar comments apply to this question as to the one at Note [3].

At this point (i.e. at the end of the transcript above) we have identified a unique position in both $\llbracket \pm \mathrm{O} \pm \Psi\left[a, b ; p\left(a_{1}\right), p\left(a_{2}\right)\right] \rrbracket$ and $\llbracket \pm \mathrm{O} \pm \Psi\left[a ; p\left(a_{1}\right), p\left(a_{2}\right), p(b)\right] \rrbracket$, i.e. in

$$
\llbracket \pm \mathrm{O} \pm\left\langle \pm\left(\begin{array}{c}
\mathrm{E}_{a} \\
\mathrm{E}_{b}
\end{array}\right) \pm\left(\begin{array}{c}
p\left(a_{1}\right) \\
p\left(a_{2}\right)
\end{array}\right)\right\rangle \rrbracket \text { and } \llbracket \pm \mathrm{O} \pm\left\langle \pm \mathrm{E}_{a} \pm\left(\begin{array}{c}
p\left(a_{1}\right) \\
p\left(a_{2}\right) \\
p(b)
\end{array}\right)\right\rangle \rrbracket
$$

This is obvious in the graphical display of Norman-G but is difficult to demonstrate succinctly here. It remains now to consider $b$ 's bringing it about that $\neg p(b)$, i.e. to determine the appropriate positions in each of $\llbracket \pm \mathrm{O} \pm \Psi[a, b ; p(b)] \rrbracket$, $\llbracket \pm \mathrm{O} \pm \Psi\left[b ; p\left(a_{1}\right), p\left(a_{2}\right), p(b)\right] \rrbracket$, and $\llbracket \pm \mathrm{O} \pm \Psi\left[a, b ; p\left(a_{1}\right), p\left(a_{2}\right), p(b)\right] \rrbracket$, i.e. in

$$
\begin{gathered}
\llbracket \pm \mathrm{O} \pm\left\langle \pm\left(\begin{array}{c}
\mathrm{E}_{a} \\
\mathrm{E}_{b}
\end{array}\right) \pm p(b)\right\rangle \rrbracket, \quad \llbracket \pm \mathrm{O} \pm\left\langle \pm \mathrm{E}_{b} \pm\left(\begin{array}{c}
p\left(a_{1}\right) \\
p\left(a_{2}\right) \\
p(b)
\end{array}\right)\right\rangle \rrbracket, \quad \text { and } \\
\llbracket \pm \mathrm{O} \pm\left\langle \pm\left(\begin{array}{c}
\mathrm{E}_{a} \\
\mathrm{E}_{b}
\end{array}\right) \pm\left(\begin{array}{c}
p\left(a_{1}\right) \\
p\left(a_{2}\right) \\
p(b)
\end{array}\right)\right\rangle \rrbracket
\end{gathered}
$$

The expressions grow too large to display side by side so henceforth we show only the questions and answers and not the asserted facts as well. The transcript continues:

$$
\begin{array}{ll}
\mathrm{P} \mathrm{E}_{b} \neg p(b) ? & -\mathrm{y} \\
\mathrm{P}\left(\neg p(b) \wedge \neg \mathrm{E}_{b} \neg p(b)\right) ? & -\mathrm{y} \\
\mathrm{P}\left(p\left(a_{1}\right) \wedge \neg \mathrm{E}_{b} p\left(a_{1}\right) \wedge \mathrm{E}_{b} \neg p(b)\right) ? & -\mathrm{y} \\
\mathrm{P}\left(p\left(a_{1}\right) \wedge \neg \mathrm{E}_{b} p\left(a_{1}\right) \wedge \neg p(b) \wedge \neg \mathrm{E}_{b} \neg p(b)\right) ? & -\mathrm{y} \\
\mathrm{P}\left(\neg p\left(a_{1}\right) \wedge \neg \mathrm{E}_{b} \neg p\left(a_{1}\right) \wedge \mathrm{E}_{b} \neg p(b)\right) ? & -\mathrm{y} \\
\mathrm{P}\left(\neg p\left(a_{1}\right) \wedge \neg \mathrm{E}_{b} \neg p\left(a_{1}\right) \wedge \neg p(b) \wedge \neg \mathrm{E}_{b} \neg p(b)\right) ? & -\mathrm{y}
\end{array}
$$

Four more assertions follow from the earlier declaration that $p\left(a_{2}\right)$ is to be treated like $p\left(a_{1}\right)$ :

$$
\mid \begin{aligned}
& \mathrm{P}\left(p\left(a_{2}\right) \wedge \neg \mathrm{E}_{b} p\left(a_{2}\right)\right.\left.\wedge \mathrm{E}_{b} \neg p(b)\right) \\
& \mathrm{P}\left(p\left(a_{2}\right) \wedge \neg \mathrm{E}_{b} p\left(a_{2}\right) \wedge \neg p(b) \wedge\right.\left.\neg \mathrm{E}_{b} \neg p(b)\right) \\
& \mathrm{P}\left(\neg p\left(a_{2}\right) \wedge \neg \mathrm{E}_{b} \neg p\left(a_{2}\right) \wedge\right.\left.\wedge \mathrm{E}_{b} \neg p(b)\right) \\
& \mathrm{P}\left(\neg p\left(a_{2}\right) \wedge \neg \mathrm{E}_{b} \neg p\left(a_{2}\right) \wedge \neg p(b) \wedge \neg \neg \mathrm{E}_{b} \neg p(b)\right)
\end{aligned}
$$

ACM Transactions on Computational Logic, Vol. 2, No. 4, October 2001. 
The system can detect can these follow from previous inputs and so does not generate questions for them. The rest of the dialogue contains just two further questions:

$$
\begin{array}{ll}
\mathrm{P}\left(\neg p\left(a_{1}\right) \wedge \neg \mathrm{E}_{b} \neg p\left(a_{1}\right) \wedge \neg p\left(a_{2}\right) \wedge \neg \mathrm{E}_{b} \neg p\left(a_{2}\right) \wedge \mathrm{E}_{b} \neg p(b)\right) ? & -\mathrm{y} \\
\mathrm{P}\left(\neg p\left(a_{1}\right) \wedge \neg \mathrm{E}_{b} \neg p\left(a_{1}\right) \wedge \neg p\left(a_{2}\right) \wedge \neg \mathrm{E}_{b} \neg p\left(a_{2}\right) \wedge \neg p(b) \wedge \neg \mathrm{E}_{b} \neg p(b)\right) ? & -\mathrm{y}
\end{array} \mid
$$

At this stage we have identified a unique position in the simplified target position (26). Identification of a position from the original target partition (24) continues in exactly the same way. Depending on the answers given, there are about a dozen or so further questions in the complete dialogue. Norman-G provides a range of features for keeping track of progress and examining which of the partitions remain to be explored. The number of questions generated in this example is further reduced if one notices that the parking of car-b is normatively independent (Section 6 ) of the parking of both $c a r-a_{1}$ and $c a r-a_{2}$. The graph can therefore be divided into independent, and much smaller, pieces. Sometimes what is normatively independent is obvious from the outset; sometimes it becomes apparent only during the course of the analysis of the example. Further development of the facilities for splitting and combining graphs in this fashion is one of the directions of current development of Norman-G.

A fuller discussion of the points arising in this dialogue, particularly those relating to the interpretation of expressions ' $x$ brings it about that', ' $x$ sees to it that', is provided in [Sergot and Richards 2000]. We limit ourselves here to one brief remark concerning the answers given at Note [3] and Note [5] of the transcript. Here we had the questions ' $\mathrm{P} \mathrm{E}_{b} p\left(a_{1}\right)$ ?' and 'P $\mathrm{E}_{b} \neg p\left(a_{1}\right)$ ?'; to keep the transcript short we answered both in the negative. Intuitively it does seem right to say that the banned administrator $b$ is not permitted to park the administrator $a$ 's car, or more precisely, to see to it or bring it about that the administrator $a$ 's car is parked. But this is not correctly represented by the expression $\neg \mathrm{P} \mathrm{E}_{b} p\left(a_{1}\right)$. For consider $\mathrm{P}\left(\mathrm{E}_{a} p\left(a_{1}\right) \wedge \mathrm{E}_{b} p\left(a_{1}\right)\right)$. It is surely permitted (in the car park that we have in mind) that the administrator $a$ and the banned administrator $b$ together act in such a way that the administrator $a$ 's car is parked in the car park. Now $\mathrm{P}(A \wedge B) \rightarrow \mathrm{P} B$ is a valid schema in the logic we are employing, and so $\vdash \mathrm{P}\left(\mathrm{E}_{a} p\left(a_{1}\right) \wedge \mathrm{E}_{b} p\left(a_{1}\right)\right) \rightarrow$ $\mathrm{P} \mathrm{E}_{b} p\left(a_{1}\right)$. It seems that we should have answered the question at Note [3] in the affirmative. (And likewise for the question at Note [5].)

Because it is so easy to slip into an erroneous reading of 'permitted to see to it that' Norman-G provides a special abbreviation: $\mathrm{P} ! \mathrm{E}_{x} F$ is intended to represent that agent $x$ is permitted to bring it about that $F$, and that this permission is not dependent on the actions of any other agents. More precisely: when the set of agents $\mathcal{A} g=\left\{x_{1}, \ldots, x_{n}\right\}$

$$
\mathrm{P} ! \mathrm{E}_{x_{i}} A \stackrel{\text { def }}{=} \mathrm{P}\left(\mathrm{E}_{x_{i}} A \wedge \neg \mathrm{E}_{x_{1}} A \wedge \ldots \wedge \neg \mathrm{E}_{x_{i-1}} A \wedge \neg \mathrm{E}_{x_{i+1}} A \wedge \ldots \wedge \neg \mathrm{E}_{x_{n}} A\right)
$$

$\mathrm{P} ! \mathrm{E}_{x}$ should here be regarded as an atomic symbol: $\mathrm{P} ! \neg \mathrm{E}_{x} F$ is not meaningful.

\section{CONCLUSION}

We have presented an extended and generalised version of the Kanger-Lindahl theory of normative positions, building upon an observation made by Makinson that 
the procedures used for generating classes of normative positions can be given a characterisation in terms of maxi-conjunctions. We used the methods to give a new account of the Kanger-Lindahl versions and showed how they can be further refined. The position generating methods and results themselves are quite general and are independent of the choice of specific deontic and action logics; in Section 5 we considered the special case where the underlying logics are those employed by Kanger and Lindahl. We showed how in that case the generation of normative positions, and associated inference methods, can be reduced to simple graph-colouring algorithms, as implemented in the Norman-G system.

The Norman-G system has been implemented in several versions in a number of programming languages. There are versions in which all nodes are stored explicitly in data structures, and versions in which only the assertions (colourings) made by the user are stored explicitly and the graph itself and colours of the nodes are computed only when they are required to be displayed. Representation of the graph in data structures and implementation of the colouring algorithms is easy; the difficult part is to devise ways of displaying selected views of the graph without overwhelming the user.

Although it is not the purpose of this paper to discuss the representational adequacy of the (extended) Kanger-Lindahl framework itself, we conclude with some brief remarks since they have a bearing on prospects for further development of Norman-G and the theory of normative positions generally.

First, as already indicated in the introduction, the framework needs to be extended to provide some treatment of (legal) 'power' ('competence'). According to the proposed characterisation in [Jones and Sergot 1996] the resulting logic would be too weak to make the generation of 'power positions' a meaningful enterprise. However, it is possible to see how some common constructions, such as (certain senses of) authorisation and some kinds of responsibility/accountability could be represented on an extended version of the Norman-G graph. These extensions will be the subject of a separate paper.

Second, for all but the simplest examples it is necessary to enrich the action component. For instance, it is one thing to say that John is permitted to bring it about that there is no fence; but it may be that John is permitted to prevent a fence being erected when there is no fence, but not permitted to remove a fence where one exists already. Extensions along these lines, which distinguish between bringing about a new state of affairs and sustaining an existing one, are the subject of current investigation. See [Sergot and Richards 2000].

Third, and most problematic, is the representation of conditional positions. It is not just the additional combinatorial complexity that must be addressed; there are also strong interactions between conditional structures and the treatment of action adopted. Introduction of a temporal component is necessary but is far from straightforward. Again, further discussion is reserved for a future paper.

A secondary aim of this paper has been to illustrate the inherent complexity of normative concepts such as duty, right, authorisation, responsibility, commitment, which are encountered not just in legal discourse, but in any description of regulated and organised agent interaction. The theory of normative positions as presented here is an important but limited component of a formal treatment of this complex network of concepts. It is already clear even from this limited theory that there is 
no point in searching for some, possibly large but nevertheless identifiable, set of basic types - 'lowest common denominators' in Hohfeld's words - in terms of which all normative relations between any (two) agents could be articulated. The representation of such relations can be taken to arbitrary levels of detail and complexity. There are nevertheless grounds to believe that a more comprehensive formal account could be developed, together with the automated support tools necessary for its practical use.

\section{ACKNOWLEDGMENTS}

This paper is dedicated to Bob Kowalski, who has been a source of inspiration and support, and a valued colleague and friend, for over 25 years. I hope that the topics of the paper are close to his heart even if the methods employed are not. The author is indebted to Andrew Jones for many valuable discussions on the topics of this paper, and to David Makinson for a number of detailed comments and suggestions on an earlier draft.

\section{REFERENCES}

Allen, L. E. And SAxon, C. S. 1986. Analysis of the logical structure of legal rules by a modernized and formalized version of Hohfeld fundamental legal conceptions. In Automated Analysis of Legal Texts, A. A. Martino and F. Socci, Eds. North-Holland, Amsterdam, 385-451.

Allen, L. E. And SAxon, C. S. 1993. A-Hohfeld: A Language for Robust Structural Representation of Knowledge in the Legal Domain to Build Interpretation-Assistance Expert Systems. In Deontic Logic in Computer Science: Normative System Specification, J.-J. C. Meyer and R. J. Wieringa, Eds. John Wiley \& Sons, Chichester, England, Chapter 8, 205-224.

ÅvVIST, L. 1974. A new approach to the logical theory of actions and causality. In Logical Theory and Semantic Analysis, S. Stenlund, Ed. Number 63 in Synthese Library. D. Reidel, Dordrecht, $73-91$.

Belnap, N. And Perloff, M. 1988. Seeing to it that: a canonical form for agentives. Theoria 54, 175-199.

Belnap, N. And Perloff, M. 1992. The way of the agent. Studia Logica 51, 463-484.

Brown, M. A. 2000. Conditional obligation and positive permission for agents in time. Nordic Journal of Philosophical Logic 5, 2 (Dec.), 83-112.

Chellas, B. F. 1969. The Logical Form of Imperatives. Dissertation, Stanford University.

Chellas, B. F. 1980. Modal Logic-An Introduction. Cambridge University Press.

Colombetti, M. 1999. Semantic, normative and practical aspects of agent communication. In Preprints of the IJCAI'g9 Workshop on Agent Communication Languages, Stockholm. 51-62.

Colombetti, M. 2000. A commitment-based approach to agent speech acts and conversations. In Proc. Workshop on Agent Languages and Conversation Policies, Autonomous Agents 2000, Barcelona.

Elgesem, D. 1992. Action Theory and Modal Logic. Doctoral thesis, Department of Philosophy, University of Oslo.

Herrestad, H. 1996. Formal Theories of Rights. Doctoral thesis, Department of Philosophy, University of Oslo.

Herrestad, H. And Krogh, C. 1995. Obligations directed from bearers to counterparties. In Proc. 5th International Conf. on Artificial Intelligence and Law, Univ. of Maryland. ACM Press, 210-218.

Hilpinen, R. 1997. On action and agency. In Logic, Action and Cognition-Essays in Philosophical Logic, E. Ejerhed and S. Lindström, Eds. Trends in Logic, Studia Logica Library, vol. 2. Kluwer Academic Publishers, Dordrecht, 3-27.

Hohfeld, W. N. 1913. Some fundamental legal conceptions as applied in judicial reasoning. Yale Law Journal 23. Reprinted with revisions as Some Fundamental Legal Conceptions as Applied 
in Judicial Reasoning, and Other Legal Essays, W. W. Cook, Ed., Yale University Press, 1919, 1923, 1964, and W. C. Wheeler, Ed., Greenwood Press, 1978.

Horty, J. F. 1996a. Agency and obligation. Synthese 108, 269-307.

Horty, J. F. 1996b. Combining agency and obligation (Preliminary version). In Deontic Logic, Agency and Normative Systems-Proc. DEON'96: 3rd International Workshop on Deontic Logic in Computer Science, Sesimbra (Portugal), M. A. Brown and J. Carmo, Eds. Workshops in Computing Series. Springer-Verlag, Berlin-Heidelberg, 98-122.

Horty, J. F. 2000. Agency and Deontic Logic. Oxford University Press.

Horty, J. F. And Belnap, N. 1995. The deliberative stit: a study of action, omission, ability, and obligation. Journal of Philosophical Logic 24, 583-644.

JENNings, N. R. 1993. Commitments and conventions: the foundation of coordination in multiagent systems. Knowledge Engineering Review 8, 3, 223-250.

Jones, A. J. I. And Sergot, M. J. 1992. Formal specification of security requirements using the Theory of Normative Positions. In Computer Security-ESORICS 92, Y. Deswarte, G. Eizenberg, and J.-J. Quisquater, Eds. Number 648 in Lecture Notes in Computer Science. Springer-Verlag, Berlin Heidelberg, 103-121.

Jones, A. J. I. And Sergot, M. J. 1993. On the Characterisation of Law and Computer Systems: The Normative Systems Perspective. In Deontic Logic in Computer Science: Normative System Specification, J.-J. C. Meyer and R. J. Wieringa, Eds. John Wiley \& Sons, Chichester, England, Chapter 12, 275-307.

Jones, A. J. I. And SERgot, M. J. 1996. A formal characterisation of institutionalised power. Journal of the IGPL 4, 3, 429-445. Reprinted in Normative Systems in Legal and Moral Theory. Festschrift for Carlos E. Alchourrón and Eugenio Bulygin, E. G. Valdés, W. Krawietz, G. H. von Wright, and R. Zimmerling, Eds. Duncker \& Humboldt, Berlin, 1997, 349-367.

Kanger, S. 1971. New foundations for ethical theory. In Deontic Logic: Introductory and Systematic Readings, R. Hilpinen, Ed. D. Reidel, Dordrecht, 36-58. Originally published as Technical Report, Stockholm University, 1957.

Kanger, S. 1972. Law and Logic. Theoria 38, 105-132.

Kanger, S. 1985. On Realization of Human Rights. In Action, Logic and Social Theory, G. Holmström and A. J. I. Jones, Eds. Acta Philosophica Fennica, Vol. 38.

Kanger, S. and Kanger, H. 1966. Rights and Parliamentarism. Theoria 32, 85-115.

Krogh, C. 1997. Normative Structures in Natural and Artificial Systems. Doctoral thesis, University of Oslo.

Lee, R. M. 1988. Bureaucracies as deontic systems. ACM Trans. Office Inf. Syst. 6, 2, 87-108.

Lindahl, L. 1977. Position and Change-A Study in Law and Logic. Number 112 in Synthese Library. D. Reidel, Dordrecht.

Lindahl, L. 1992. Stig Kanger's Theory of Rights. In 9th International Congress of Logic, Methodology and Philosophy of Science. Stig Kanger Memorial Symposium on the Logic of Rights and Choices, Uppsala.

MAKINSON, D. 1986. On the formal representation of rights relations. Journal of Philosophical Logic 15, 403-425.

Perloff, M. 1991. 'Stit' and the language of agency. Synthese 86, 379-408.

Pörn, I. 1970. The Logic of Power. Blackwells, Oxford.

Pörn, I. 1974. Some basic concepts of action. In Logical Theory and Semantic Analysis, S. Stenlund, Ed. Number 63 in Synthese Library. D. Reidel, Dordrecht, 93-101.

Pörn, I. 1977. Action Theory and Social Science: Some Formal Models. Number 120 in Synthese Library. D. Reidel, Dordrecht.

PörN, I. 1989. On the nature of a social order. In Logic, Methodology and Philosophy of Science VIII, J. E. Fenstad et al., Eds. Elsevier Science Publishers, 553-567.

Santos, F. and Carmo, J. 1996. Indirect action, influence and responsibility. In Deontic Logic, Agency and Normative Systems-Proc. DEON'96: 3rd International Workshop on Deontic Logic in Computer Science, Sesimbra (Portugal), M. A. Brown and J. Carmo, Eds. Workshops in Computing Series. Springer-Verlag, Berlin-Heidelberg, 194-215. 
Santos, F., Jones, A. J. I., And Carmo, J. 1997. Action concepts for describing organised interaction. In Proc. 13th Annual Hawaii International Conf. on System Sciences. Vol. V. IEEE Computer Society Press, Los Alamitos, California.

Segerberg, K. 1985. Routines. Synthese 65, 185-210.

Segerberg, K. 1989. Bringing it about. Journal of Philosophical Logic 18, 327-347.

Segerberg, K. 1992. Getting started: Beginnings in the logic of action. Studia Logica 51, $347-378$.

Sergot, M. J. 1996. A Computational Theory of Normative Positions. II Non-regular logics. Tech. rep., Department of Computing, Imperial College. Jan.

SERGOT, M. J. AND Richards, F. M. 2000. On the representation of action and agency in the theory of normative positions. In Proc. DEON'00: 5th International Workshop on Deontic Logic in Computer Science, Toulouse, R. Demolombe and R. Hilpinen, Eds. To appear in Fundamenta Informaticae.

Shонам, Y. 1991. Implementing the intentional stance. In Philosophy and AI: Essays at the Interface, R. Cummins and J. Pollock, Eds. MIT Press, Cambridge, Mass., 261-277.

Sнонам, Y. 1993. Agent-oriented programming. Artificial Intelligence 60, 51-92.

Singh, M. P. 1998. Agent communication languages: Rethinking the principles. IEEE Computer 31, 40-47.

Singh, M. P. 1999. A social semantics for agent communication languages. In Preprints of the IJCAI'99 Workshop on Agent Communication Languages, Stockholm. 75-88.

TALja, J. 1980. A technical note on Lars Lindahl's Position and Change. Journal of Philosophical Logic 9, 167-183.

Ting, T. C. 1990. Application information security semantics: A case of mental health delivery. In Database Security: Status and Prospects III, D. L. Spooner and C. E. Landwehr, Eds. NorthHolland, Amsterdam.

Received November 2000; revised April 2001; accepted April 2001 